\title{
Neglected X-ray discovered polars
}

\section{The peculiar eclipsing binary HY Eridani}

\author{
K. Beuermann ${ }^{1}$, V. Burwitz ${ }^{2}$, K. Reinsch ${ }^{1}$, A. Schwope ${ }^{3}$, and H.-C. Thomas ${ }^{4, \star}$ \\ ${ }^{1}$ Institut für Astrophysik, Georg-August-Universität, Friedrich-Hund-Platz 1, 37077 Göttingen, Germany \\ e-mail: k. beuermann@t-online.de \\ 2 MPI für Extraterrestrische Physik, Giessenbachstr. 6, 85740 Garching, Germany \\ ${ }^{3}$ Leibniz-Institut für Astrophysik Potsdam (AIP), An der Sternwarte 16, 14482 Potsdam, Germany \\ ${ }^{4}$ MPI für Astrophysik, Karl-Schwarzschild-Str. 1, 85740 Garching, Germany \\ Received 3 September 2019 / Accepted 2 January 2020
}

\begin{abstract}
We report on the X-ray observations of the eclipsing polar HY Eri (RX J0501-0359), along with its photometric, spectrophotometric, and spectropolarimetric optical variations, collected over 30 years. With an orbital period of $2.855 \mathrm{~h}$, HY Eri falls near the upper edge of the 2-3 h period gap. After 2011, the system went into a prolonged low state, continuing to accrete at a low level. We present an accurate alias-free long-term orbital ephemeris and report a highly significant period change by $10 \mathrm{~ms}$ that took place over the time interval from 2011 to 2018 . We acquired a high-quality eclipse spectrum that shows the secondary star as a dM5-6 dwarf at a distance $d=1050 \pm 110 \mathrm{pc}$. Based on phase-resolved cyclotron and Zeeman spectroscopy, we identify the white dwarf (WD) in HY Eri as a two-pole accretor with nearly opposite accretion spots of 28 and 30 MG. The Zeeman analysis of the low state spectrum reveals a complex magnetic field structure, which we fit by a multipole model. We detected narrow emission lines from the irradiated face of the secondary star, of which $\mathrm{Mg} \mathrm{I} \lambda 5170$ with a radial velocity amplitude of $K_{2}^{\prime}=139 \pm 10 \mathrm{~km} \mathrm{~s}^{-1}$ (90\% confidence) tracks the secondary more reliably than the narrow $\mathrm{H} \alpha$ line. Based on the combined dynamical analysis and spectroscopic measurement of the angular radius of the WD, we obtain a primary mass of $M_{1}=0.42 \pm 0.05 M_{\odot}$ (90\% confidence errors), identifying it as a probable He WD or hybrid HeCO WD. The secondary is a main sequence star of $M_{2}=0.24 \pm 0.04 M_{\odot}$ that seems to be slightly inflated. The large distance of HY Eri and the lack of similar systems suggest a very low space density of polars with low-mass primary. According to current theory, these systems are destroyed by induced runaway mass transfer, suggesting that HY Eri may be doomed to destruction. Over the last 30 years, HY Eri experienced high and low states with mass transfer rates that differed by three orders of magnitude, varying between $\dot{M} \simeq 10^{-9} M_{\odot} \mathrm{yr}^{-1}$ and $10^{-12} M_{\odot} \mathrm{yr}^{-1}$. At a galactic latitude of $-26.1^{\circ}$, it is located about $500 \mathrm{pc}$ below the galactic plane.
\end{abstract}

Key words. novae, cataclysmic variables - white dwarfs - binaries: close - binaries: eclipsing - stars: magnetic field stars: individual: HY Eridani

\section{Introduction}

Of the more than 1200 cataclysmic variables in the final 2016 edition of the Ritter \& Kolb (2003) catalog, 114 are confirmed polars (or AM Herculis binaries), which contain a late-type main sequence star and an accreting magnetic white dwarf in synchronous rotation. The name "polar" was coined by Krzeminski \& Serkowski (1977) to describe the high degree of circular polarization, which became one of the hallmarks of the class. Another is the large portion of the bolometric luminosity emitted in high states of accretion in form of soft and hard X-ray emission, which led to the discovery of the majority of the known systems. Many individual polars are characterized by idiosyncrasies, which distinguish them from their peers and provide special insight into the physics of polars. Unresolved questions relate, for example, to the physics of accretion (Bonnet-Bidaud et al. 2000, 2015; Busschaert et al. 2015), various aspects of close-binary evolution (Webbink \& Wickramasinghe 2002; Liebert et al. 2005; Knigge et al. 2011), and the generation and structure of the magnetic field of the white dwarf (WD; Beuermann et al. 2007; Wickramasinghe et al. 2014; Ferrario et al. 2015).

Our optical programs for identifying high-galactic latitude ROSAT X-ray sources (Thomas et al. 1998; Beuermann et al.

\footnotetext{
$\star$ Deceased 18 January 2012 .
}

1999; Schwope et al. 2002) have led to the discovery of 27 new polars. Twenty sources have been described in previous publications. In this series of three papers, we present results on the remaining seven. Paper I (Beuermann et al. 2017) describes V358 Aqr (=RX J2316-05), a system that experiences giant flares on its secondary star. Here we present a comprehensive analysis of the eclipsing polar HY Eri (=RX J0501-03) based on data collected over three decades. Our early conference paper (Burwitz et al. 1999) represents the only previous account of the system in the literature. The third paper of this series will contain shorter analyses of RX J0154-59, RX J0600-27, RX J0859+05, RX J0953+14, and RX J1002-19, of which three have not been addressed previously either.

\section{Observations}

\subsection{X-ray data}

HY Eri, located at $\mathrm{RA}(2000)=05^{\mathrm{h}} 01^{\mathrm{m}} 46^{\prime \prime} \cdot 4, \quad \operatorname{Dec}(2000)=$ $-03^{\circ} 59^{\prime} 20^{\prime \prime}(l, b=203.5,-26.1)$ was discovered as a very soft X-ray source in the RASS (Boller et al. 2016) ${ }^{1}$ and identified by us spectroscopically with an eclipsing polar

\footnotetext{
1 http://cdsarc.u-strasbg.fr/viz-bin/qcat?J/A+A/588/ A103
} 
Table 1. Time-resolved X-ray observations of HY Eri.

\begin{tabular}{lllrlll}
\hline \hline Dates & Instrument & $\begin{array}{c}\text { Band } \\
(\mathrm{keV})\end{array}$ & $\begin{array}{r}\text { Total } \\
(\mathrm{ks})\end{array}$ & State & Ref. \\
\hline 24-26 Aug 1990 & RASS PSPC & $0.1-2$ & 0.5 & High & $(1)$ \\
24 Feb 1992 & ROSAT PSPC & $0.1-2$ & 2.5 & High & $(1)$ \\
15-22 Feb 1993 & ROSAT PSPC & $0.1-2$ & 1.9 & High & $(1)$ \\
8-16 Sep 1995 & ROSAT HRI & $0.1-2$ & 16.5 & High & $(1)$ \\
26 Feb-19 Mar 1996 & ROSAT HRI & $0.1-2$ & 28.8 & High & $(1)$ \\
24 Mar 2002 & XMM MOS+pn & $0.2-10$ & 6.6 & High & $(2)$ \\
\hline
\end{tabular}

References. (1) Burwitz et al. (1999), (2) this work.

(Beuermann \& Thomas 1993; Beuermann et al. 1999; Burwitz et al. 1999). Follow-up pointed ROSAT observations were performed 1992 and 1993 with the Position Sensitive Proportional Counter (PSPC) as the detector and 1995 and 1996 with the High Resolution Imager (HRI). These data were originally published by Burwitz et al. (1999) and reanalyzed for the present study. We also analyzed the previously unpublished data taken in 2002 with $X M M-N e w t o n$ equipped with the EPIC camera. On all occasions, HY Eri was encountered with an X-ray flux that corresponds to a high or near high state ${ }^{2}$ (Table 1 ).

\subsection{Optical photometry}

Orbital $B V R I$ light curves and $V$-band eclipse light curves were measured in 1994 and 1996 with the ESO/Dutch 0.9 m telescope (Burwitz et al. 1999). Extensive white-light (WL) photometry, performed between 2010 and 2019 with the $1.2 \mathrm{~m} \mathrm{MONET/N}$ and MONET/S telescopes at the McDonald Observatory and the South African Astrophysical Observatory, respectively, allowed us to establish an alias-free long-term ephemeris. Seven-color grizJHK photometry was performed with the MPG/ESO $2.2 \mathrm{~m}$ telescope equipped with the GROND ${ }^{3}$ photometer in 2016, 2017, and 2018. A $\log$ of the observations is provided in Table 2. We measured magnitudes relative to the dM1-2 star SDSS 050146.02-040042.2 (referred to as C1), which is located $43^{\prime \prime} \mathrm{E}$ and $4^{\prime \prime} \mathrm{N}$ of HY Eri and has Sloan AB magnitudes $g=$ $16.91, r=16.38, i=16.23$, and $z=16.17$. Its color, $g-i=0.68$, is similar to the low-state Sloan color of HY Eri, $g-i=0.71$. HY Eri is separated by only 6 ". 2 from the center of a galaxy with Sloan $r=18.44$. All accepted eclipse light curves were taken in sufficiently good seeing to escape spillover from the galaxy.

\subsection{Optical spectroscopy}

Follow-up time-resolved spectrophotometric observations of HY Eri in its high state were performed in 1993 and 1995, using the ESO $1.5 \mathrm{~m}$ telescope with the Boller \& Chivens spectrograph and the ESO/MPG $2.2 \mathrm{~m}$ telescope with EFOSC2, respectively. In the latter run, grisms G1 and G3 yielded low- and mediumresolution spectra with FWHM resolutions of $25 \AA$ and $8 \AA$ that covered the entire optical band and the blue band, respectively.

\footnotetext{
2 Photometrically, "high" and "low" states refer to two brightness levels between which polars oscillate in their long-term light curves. Spectroscopically, a "high" state is usually characterized by intense He II $\lambda 4686$ line emission, which is absent in a "low" state. Physically, "high" refers to accretion rates adequate to drive the standard secular evolution of CVs, while in a "low" state, accretion ceases either completely or is reduced to a trickle. "Intermediate" refers to temporary states in between.

3 Gamma-ray Burst Optical/Near-infrared Detector.
}

Table 2. Journal of time-resolved optical photometry.

\begin{tabular}{lrccccc}
\hline \hline Dates & $\begin{array}{c}\text { Number } \\
\text { nights }\end{array}$ & Band & $\begin{array}{c}\text { Expos. } \\
(\mathrm{s})\end{array}$ & $\begin{array}{c}\text { Total } \\
(\mathrm{h})\end{array}$ & State & Tel. \\
\hline Feb 1994, Jan 1996 & 3 & $\mathrm{~V}$ & $20 / 60$ & 11.7 & High & $(1)$ \\
3-5 Feb 2001 & 2 & R,Gunn i & 60 & 1.1 & High & $(2)$ \\
17-22 Jan 2010 & 5 & WL & $10 / 60$ & 10.6 & High & $(3)$ \\
8-18 Nov 2010 & 3 & WL & $10 / 60$ & 4.2 & Interm. & $(3)$ \\
Feb-Oct2011 & 3 & WL & $10 / 60$ & 3.6 & Interm. & $(3)$ \\
Aug 2014-Jan 2015 & 10 & WL & $15 / 60$ & 8.0 & Low & $(3)$ \\
24/27 Oct 2016 & 2 & grizJHK & 35 & 5.1 & Interm. & $(4)$ \\
Sep 2017-Jan 2019 & 23 & WL & $15 / 60$ & 34.8 & Low & $(5)$ \\
Nov 2017 & 2 & grizJHK & 35 & 4.7 & Low & $(4)$ \\
Feb 2018 & 1 & grizJHK & 35 & 3.0 & Low & $(4)$ \\
\hline
\end{tabular}

Notes. (1) ESO/Dutch $0.9 \mathrm{~m}$, (2) ESO $3.6 \mathrm{~m}$ with EFOSC2, (3) McD, MONET/N $1.2 \mathrm{~m}$, (4) MPG/ESO $2.2 \mathrm{~m}$ with GROND, (5) SAAO, MONET/S $1.2 \mathrm{~m}$.

Table 3. Time-resolved spectroscopy and circular spectropolarimetry.

\begin{tabular}{lcccccccc}
\hline \hline Dates & $\begin{array}{c}\text { Band } \\
(\AA)\end{array}$ & $\begin{array}{c}\text { Resol. } \\
(\AA)\end{array}$ & $\begin{array}{c}\text { Number } \\
\text { Expos. Total } \\
(\mathrm{min})\end{array}$ & State & Instr. \\
\hline 13-17 Dec 1993 & $3500-9500$ & 25 & 50 & 10.0 & 10.6 & High & $(1)$ \\
14 Nov 1995 & $3800-9119$ & 25 & 24 & 2.0 & 1.1 & High & $(2)$ \\
15 Nov 1995 & $3869-5109$ & 8 & 17 & 10.0 & 3.2 & High & $(2)$ \\
20 Nov 2000 & $6340-10400$ & 10 & 8 & 0.5 & 0.5 & High & $(3)$ \\
20 Nov 2000 & $6340-10400$ & 10 & 1 & 10.0 & & & $(3)$ \\
31 Dec 2008 & $3800-9200$ & 10 & 40 & 6.5 & 5.6 & Low & $(4)$ \\
\hline
\end{tabular}

Notes. (1) ESO $1.5 \mathrm{~m}, \mathrm{~B} \& \mathrm{C}$ spectrograph, (2) MPG/ESO $2.2 \mathrm{~m}$ with EFOSC2, (3) ESO VLT UT1 with FORS1, (4) ESO VLT UT2 with FORS1.

Using the photometrically established eclipse ephemeris, a $10 \mathrm{~min}$ exposure in the near-total eclipse was taken with the ESO/VLT UT1 equipped with FORS 1 on 20 November 2000. Grating G300I provided coverage of the red part of the spectrum, which is dominated by the secondary star of HY Eri. The magnetic nature of the WD was studied in 2008 by phase-resolved low-resolution circular spectropolarimetry performed with the ESO/VLT UT2 and FORS1. Grism G300V provided coverage from 3800 to $9200 \AA$. Table 3 lists the wavelength ranges, number of spectra, exposure times, and total times spent on source.

\subsection{Synthetic white light photometry}

As described in Paper I, we performed synthetic photometry in order to tie the WL measurements obtained with the MONET telescopes into the standard ugriz system. We defined a MONETspecific WL AB magnitude $w$, which has its pivot wavelength $\lambda_{\text {piv }}=6379 \AA$ in the red part of the Sloan $r$ band. For a star with the colors of comparison star $\mathrm{C} 1$, the color difference is $w-r=+0.07$. Hence, unity relative WL flux corresponds to $r=16.38$ and $w=16.45$. For WL measurements of other stars, $w$ is a measured quantity and is related to Sloan $r$ by $r=w-$ $(w-r)_{\text {syn }}$. For a wide range of incident spectra, the synthetic color $\left|(w-r)_{\text {syn }}\right| \lesssim 0.1$. Hence, $w \simeq r$ is typically correct within $0.1 \mathrm{mag}$, except for very red stars.

\section{Optical light curves}

\subsection{Orbital light curves}

In Fig. 1, we show the $V$-band and WL light curves in the high states of 11 January 1996 and 19 January 2010, respectively, 

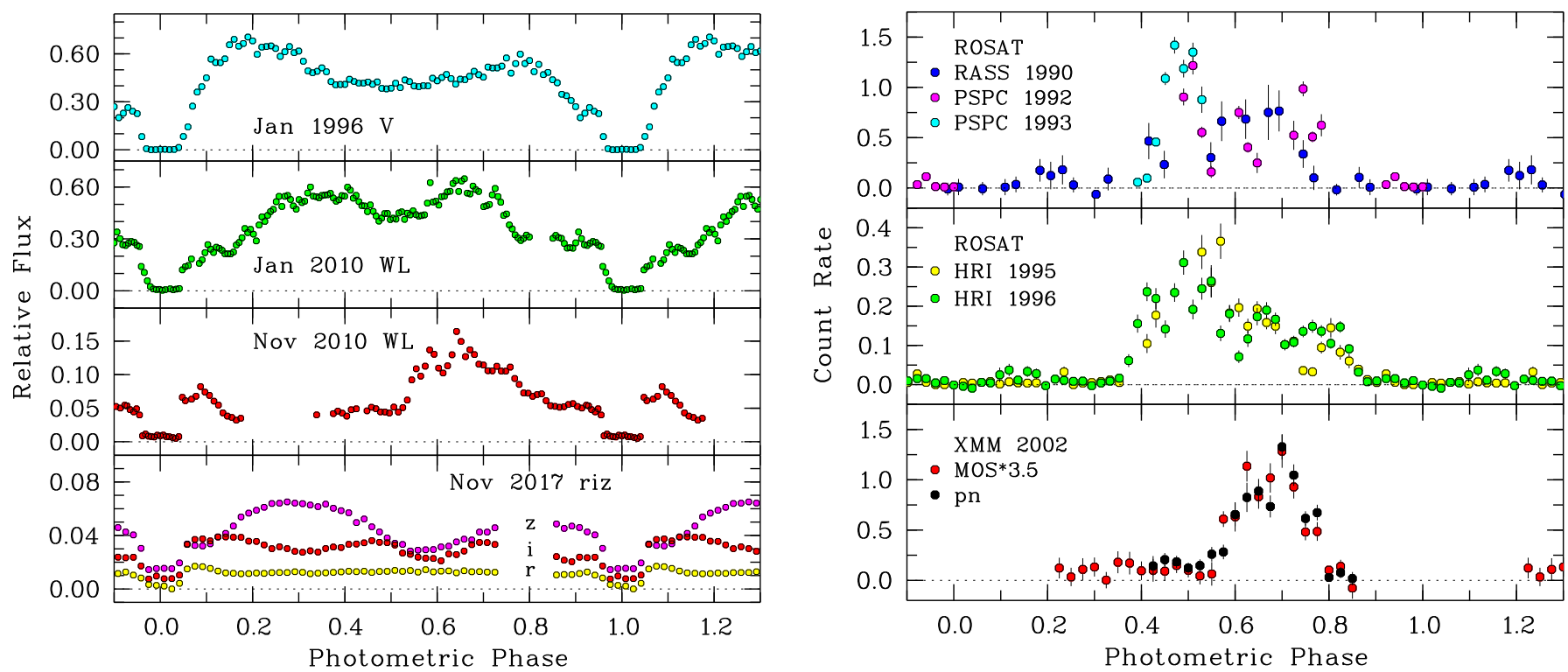

Fig. 1. Left, from top: orbital light curves of HY Eri in the 1996 and January 2010 high states, November 2010 intermediate, and 2017 low state, binned to $\sim 2$ min time resolution. Right, from top: binned ROSAT X-ray light curves measured with either the PSPC or the HRI as detectors and $X M M-N e w t o n$ light curve measured with the MOS and pn detectors of the EPIC camera.
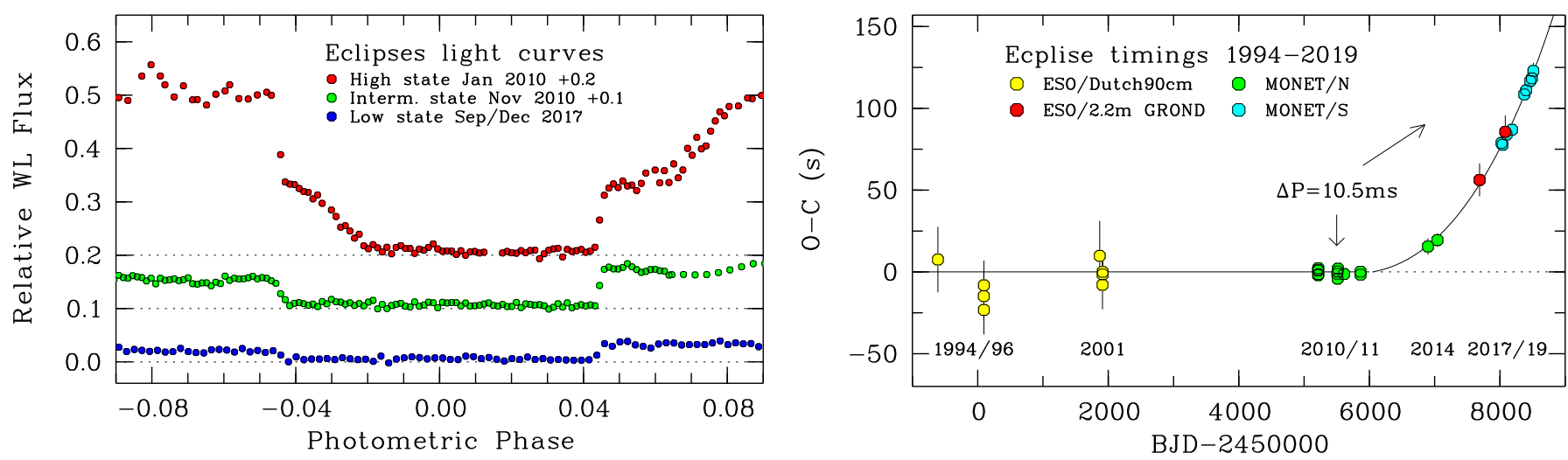

Fig. 2. Left: eclipse light curves in the high, intermediate, and in the low state, the former two shifted upward to avoid overlap. Phases are from Eq. (1) for the high and intermediate states and from and Eq. (2) for the low state. Right: $O-C$ diagram for the deviations of the mid-eclipse times from the linear ephemeris of Eq. (1), showing the change in orbital period.

the WL light curve in the intermediate state of 14-18 November 2010, and the riz low-state light curves of 24-25 November 2017. Orbital phase $\phi=0$ is defined by the eclipse ephemeris provided in Sect. 3.3. HY Eri reached orbital maxima of $V=16.8$ and $w=17.1$ in the high states and $w=18.7$ in the intermediate state. The 2017 peak magnitudes were $z=19.1$ and $i=19.7$, while $r$ stayed at 21 throughout the orbit outside eclipse. In all states, the light curves exhibit the signatures of emission from two accretion regions, being shaped by cyclotron beaming. The same holds for the light curves in the right panel of Fig. 6. Borrowing from the insight provided by the low-state spectropolarimetry (Sect. 6.1), we identify, for instance, the double-humped $z$-band light curve in the lower left panel of Fig. 1 as cyclotron emission in the 4th harmonic from two accretion regions best viewed at phases $\varphi \simeq 0.35$ and 0.85 . The light curves in WL are less easily interpreted because of the lack of wavelength resolution. We loosely refer to the emission regions best seen around $\phi=0.85$ and $\phi \simeq 0.35$ as "pole 1 " and "pole 2 " or "spot 1 " and "spot 2", respectively. In the high state, the emissions from both poles become an inextricable conglomerate. The available evidence suggests that HY Eri is a permanent two-pole emitter.

\subsection{Eclipse light curves}

We collected a total of 41 eclipses of the hot spot on the WD by the secondary star, 13 in various high states, eight in intermediate states, and 20 in low states. Ingress and egress of the hot spot take place in typically less than 20 s. In Fig. 2, we show the eclipse light curves in the three states at the original time resolutions of $13 \mathrm{~s}$ (exposure and readout) for the high and intermediate states and and $22 \mathrm{~s}$ for the low state. A characteristic feature of the high state is the delayed eclipse of the accretion stream. This component disappears, when the accretion rate drops.

The eclipse was modeled by the occultation of a circular disk of uniform surface brightness, which represents either the WD or the hot spot on the WD. The parameters of the fit are the mideclipse time, the FWHM, and the duration of ingress or egress. We improved the statistics and reduced the timing error in the low state, when the star became as faint as $w=20.7$ outside eclipse, by fitting up to $n=4$ eclipses on a barycentric time scale if taken nearby in time. The resulting mid-eclipse times are listed in Table 4 . In the cases with $n>1$, the cycle number of the bestdefined eclipse is quoted. The measured FWHM of the eclipse is 
Table 4. Observed mid-eclipse times of HY Eri.

\begin{tabular}{|c|c|c|c|c|c|c|c|c|}
\hline Cycle & $\begin{array}{l}\text { BJD(TDB) } \\
2400000+\end{array}$ & $\mathrm{n}$ & $\begin{array}{r}\text { Error } \\
(\mathrm{s})\end{array}$ & $\begin{array}{r}O-C \\
(\mathrm{~s})\end{array}$ & State & $\begin{array}{c}\text { Expos } \\
\text { (s) }\end{array}$ & Band & Instr. \\
\hline-54428 & 49390.622004 & 1 & 20.0 & 7.6 & High & 20 & $V$ & (1) \\
\hline-48518 & 50093.728987 & 1 & 15.0 & -14.8 & High & 60 & $V$ & (1) \\
\hline-48511 & 50094.561847 & 1 & 15.0 & -8.2 & High & 30 & V & (1) \\
\hline-48510 & 50094.680642 & 1 & 15.0 & -23.2 & High & 30 & $V$ & (1) \\
\hline-33598 & 51868.747892 & 1 & 21.5 & 9.8 & High & 60 & Spec & (2) \\
\hline-33229 & 51912.647277 & 1 & 15.0 & -7.9 & High & 60 & $R$ & (3) \\
\hline-33213 & 51914.550874 & 1 & 10.0 & 0.1 & High & 60 & $R$ & (3) \\
\hline-33212 & 51914.669824 & 1 & 10.0 & -1.5 & High & 60 & Gunn i & (3) \\
\hline-5483 & 55213.563377 & 1 & 2.1 & 1.3 & High & 10 & WL & (4) \\
\hline-5474 & 55214.634061 & 1 & 3.2 & -2.0 & High & 10 & WL & (4) \\
\hline-5465 & 55215.704814 & 1 & 1.2 & 0.8 & High & 10 & WL & (4) \\
\hline-5449 & 55217.608337 & 1 & 1.2 & 2.3 & High & 10 & WL & (4) \\
\hline-5439 & 55218.797986 & 1 & 2.6 & -1.4 & High & 10 & WL & (4) \\
\hline-3000 & 55508.963566 & 1 & 2.2 & -1.1 & Interm. & 10 & WL & (4) \\
\hline-2959 & 55513.841263 & 1 & 1.8 & -4.1 & Interm. & 10 & WL & (4) \\
\hline-2958 & 55513.960283 & 1 & 1.2 & 0.3 & Interm. & 10 & WL & (4) \\
\hline-2916 & 55518.957004 & 1 & 1.5 & 2.0 & Interm. & 10 & WL & (4) \\
\hline-2129 & 55612.585630 & 1 & 2.8 & -1.3 & Interm. & 10 & WL & (4) \\
\hline-17 & 55863.848336 & 1 & 2.1 & 0.2 & Interm. & 10 & WL & (4) \\
\hline 0 & 55865.870789 & 1 & 1.1 & -1.7 & Interm. & 10 & WL & (4) \\
\hline 8675 & 56897.927726 & 3 & 5.0 & 15.6 & Low & 20 & WL & (4) \\
\hline 9892 & 57042.713137 & 1 & 4.2 & 19.5 & Low & 15 & WL & (4) \\
\hline 15315 & 57687.882864 & 1 & 10.0 & 56.2 & Interm. & 33 & $\mathrm{iz}$ & (5) \\
\hline 18137 & 58023.613860 & 1 & 1.7 & 78.9 & Low & 15 & WL & (6) \\
\hline 18279 & 58040.507455 & 1 & 2.8 & 77.7 & Low & 15 & WL & (6) \\
\hline 18635 & 58082.860538 & 1 & 10.0 & 85.6 & Low & 33 & $\mathrm{iz}$ & (5) \\
\hline 18800 & 58102.490416 & 1 & 4.6 & 84.0 & Low & 15 & WL & (6) \\
\hline 19496 & 58185.292928 & 2 & 3.8 & 86.9 & Low & 15 & WL & (6) \\
\hline 21070 & 58372.550511 & 4 & 3.0 & 109.1 & Low & 15 & WL & (6) \\
\hline 21322 & 58402.530740 & 3 & 4.2 & 111.0 & Low & 15 & WL & (6) \\
\hline 21835 & 58463.561939 & 2 & 2.9 & 116.4 & Low & 15 & WL & (6) \\
\hline 22237 & 58511.387633 & 1 & 6.9 & 127.2 & Low & 15 & WL & (6) \\
\hline
\end{tabular}

Notes. (1) ESO/Dutch 0.9 m, (2) ESO VLT UT1, EFOSC 1, (3) ESO $3.6 \mathrm{~m}$, EFOSC 2, (4) McD MONET/N 1.2 m, (5) MPG/ESO $2.2 \mathrm{~m}$ with GROND, (6) SAAO MONET/S $1.2 \mathrm{~m}$.

the same for the different accretion states with a mean value of $\Delta t_{\mathrm{ecl}}=910.6 \pm 1.5 \mathrm{~s}$ or $\Delta \phi=0.08859 \pm 0.00015$ in phase units. This is the longest relative eclipse width of all polars. We did not detect the ingress and egress of the WD photosphere because our WL observations are dominated by cyclotron emission and the measurements in the GROND $g r$ filters lacked the required time resolution. The quoted mid-eclipse times refer to the hot spot on the WD and may deviate from true inferior conjunction of the secondary by up to $\sim 30 \mathrm{~s}$ or $\sim 0.003$ in phase.

The relative WL flux in the totality is the same in the high, intermediate, and low states, with a mean of $0.0060 \pm 0.0005$ or $w=22.0 \pm 0.1$. Using a color correction $w-r \simeq-0.3$, appropriate for the secondary star, we find $r \simeq 22.3$, which compares favorably with the result of our spectrophotometry in eclipse reported Sect. 5.2. Hence, the residual WL flux in eclipse is largely that of the secondary star.

\subsection{Eclipse ephemeris}

We corrected the UTC eclipse times to Barycentric Dynamical Time (TDB), using the tool provided by Eastman et al. $(2010)^{4}$, which accounts also for the leap seconds. The complete set of eclipse times is presented in Table 4. The 2010 and 2011 data

\footnotetext{
4 http://astroutils.astronomy.ohio-state.edu/time/
}

Table 5. Parameters of X-ray spectral fits for HY Eri.

\begin{tabular}{lccccc}
\hline \hline Observation & Detector & $T_{\mathrm{bb}}$ & $N_{\mathrm{H}}$ & $F_{\mathrm{bb}}$ & $F_{\mathrm{th}}$ \\
\cline { 5 - 6 } & & $(\mathrm{eV})$ & $\left(10^{20} \mathrm{~cm}^{-2} \mathrm{~s}\right)$ & $\left(10^{-11} \mathrm{erg} \mathrm{cm}^{-2} \mathrm{~s}\right)$ \\
\hline RASS 1990 & PSPC & $30:$ & 3.0 fixed & $9:$ & \\
ROSAT 1992/93 & PSPC & $37 \pm 6$ & $8.0 \pm 2.0$ & $43 \pm 30$ & \\
& & $44 \pm 3$ & 6.0 fixed & 13.0 & \\
& & $50 \pm 3$ & 3.0 fixed & 1.7 & \\
XMM 2002 & MOS1+pn & $45 \pm 6$ & $1.4+2.0,-1.4$ & 0.5 & 0.08 \\
& & $40 \pm 2$ & 3.0 fixed & 1.5 & 0.09 \\
\hline
\end{tabular}

and the scanty earlier data define the alias-free linear ephemeris

$T_{\mathrm{ecl}}=\mathrm{BJD}(\mathrm{TDB}) 2455865.87081(1)+0.118969076(2) E$,

with $\chi^{2}=19.6$ for 18 degrees of freedom (solid line, cycle numbers $E \leq 0$ ). The $O-C$ offsets from the ephemeris of Eq. (1) are displayed in Fig. 2, right panel. This ephemeris became increasingly invalid after 2011 and the more recent data are well represented by a cubic ephemeris for $E>-6000$ (solid curve). Currently, $O-C$ relative to the linear ephemeris of Eq. (1) has exceeded $2 \mathrm{~min}$, which is much too large to be explained by wandering of the accretion spot. The mid-eclipse times of 2017 to 2019 follow the linear ephemeris

$T_{\mathrm{ecl}}=\mathrm{BJD}(\mathrm{TDB}) 2455865.86951(14)+0.118969198(8) E$,

implying a period change relative to Eq. (1) of $10.5 \pm 0.7 \mathrm{~ms}$. The mean rate of the period variation between 2011 and 2018 is $\dot{P} \simeq 5 \times 10^{-11} \mathrm{ss}^{-1}$. The period change started approximately, when the system entered a prolonged low state after 2011. This is likely a coincidence, however, because it was also in a low state in 2008 and temporarily in an intermediate state in 2016.

\section{X-ray light curves and spectra}

The ROSAT soft X-ray light curves taken between 1990 and 1996 (Burwitz et al. 1999) show a structured bright phase that extends from $\phi \simeq 0.4$ to $\phi \simeq 0.8$ with low-level emission over the remainder of the orbit. Binned versions of these data are shown in Fig. 1, right panels. We also included the previously unpublished light curve measured with XMM-Newton and the MOS and pn detectors of the EPIC camera in 2002. The lower right panel of Fig. 1 shows the mean count rates of the two MOS detectors and the pn-detector, respectively, with the former adjusted by a factor of 3.5 upward. The X-ray bright phases in the ROSAT and XMM-Newton light curves show some similarity with the WL optical light curve of November 2010, suggesting that the observed intense X-ray emission originates from pole 1, phase-modulated by rotation of the WD and possibly by internal absorption. The low-level emission around $\phi=0.3$ may stem from pole 2 . The bright phase reached count rates around 1.0 PSPC cts s${ }^{-1}, 0.4$ HRI cts s ${ }^{-1}$, and 1 EPIC-pn cts s ${ }^{-1}$, suggesting that HY Eri was in some form of high or intermediate-to-high state during these observations.

The ROSAT and XMM-Newton spectra (not shown) are characterized by an intense soft X-ray and an underlying hard X-ray component, of which the latter is well covered only in the $X M M$-Newton run. We fitted the bright-phase spectra with the sum of a blackbody of temperature $k T_{\mathrm{bb}}$ and a thermal component with the temperature fixed at $10 \mathrm{keV}$, both attenuated by a column density $N_{\mathrm{H}}$ of cold interstellar matter of solar composition ${ }^{5}$. The fit parameters are listed in Table 5. The values

\footnotetext{
5 Using XSPEC version 12.9.1n with TBabs*(bbody+apec).
} 

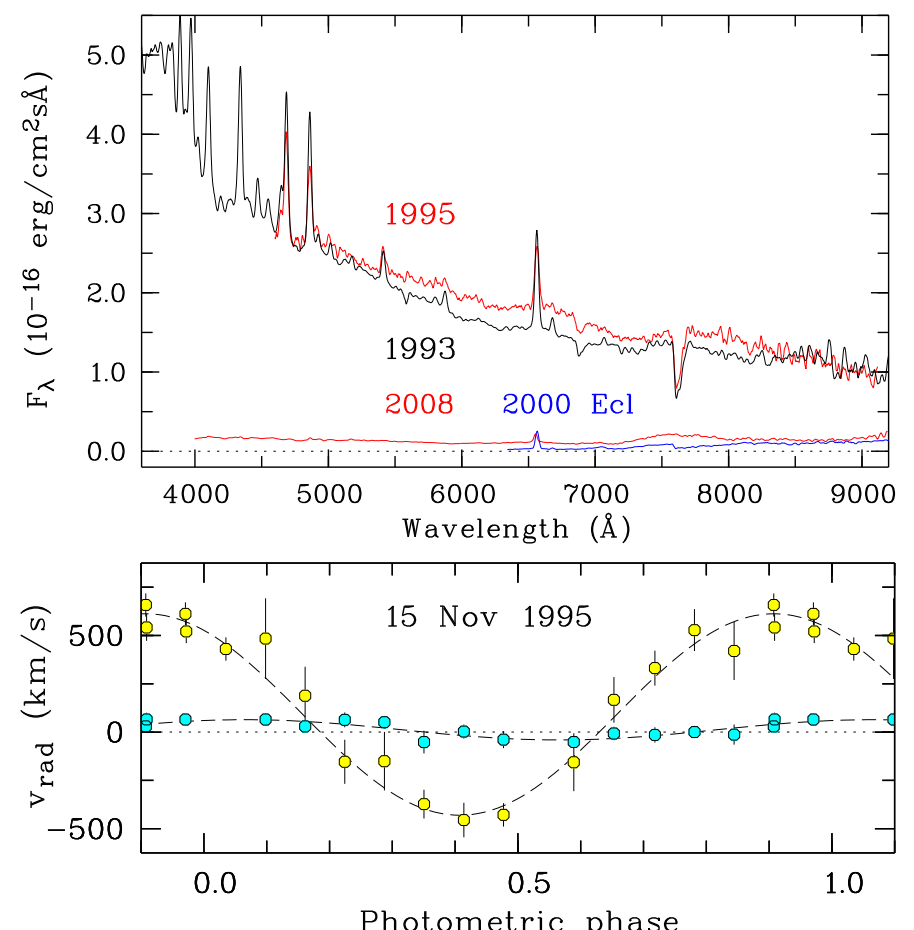

Fig. 3. Top: mean flux-calibrated low-resolution spectra of HY Eri in the high states of 1993 and 1995. For comparison, the eclipse spectrum of 2000 (blue curve) and the mean out-of-eclipse spectrum in the 2008 low state (red curve) are added on the same ordinate scale. Bottom: mean radial velocities of the broad emission lines of $\mathrm{H} \beta$ and HeII $\lambda 4686$ (open circles) and of the line peaks (cyan dots) derived from mediumresolution spectra taken on 15 November 1995.

of $k T_{\mathrm{bb}}$ and $N_{\mathrm{H}}$ differ substantially, indicating either true variability or systematic uncertainties caused by the poor energy resolution of the ROSAT PSPC, the lack of spectral coverage of the XMM-Newton detectors below $0.2 \mathrm{keV}$, or the inadequacy of fitting a multi-temperature source by a single blackbody (Beuermann et al. 2012). In any case, HY Eri is more strongly absorbed than other polars. For the combined PSPC fit to the 1992 and 1993 data, $N_{\mathrm{H}}$ exceeds the total galactic column density $N_{\mathrm{H}, \mathrm{gal}} \simeq 5.2 \times 10^{20} \mathrm{H}$-atoms $\mathrm{cm}^{-2}$ at the position of HY Eri (Hi4PI Collaboration 2016) ${ }^{6}$. The galactic extinction at the position of HY Eri, $A_{V}=0.172$ (Schlegel et al. 1998), and the $N_{\mathrm{H}}-A_{V}$ conversion factor of Predehl \& Schmitt (1995) yield $N_{\mathrm{H}} \simeq 3 \times 10^{20} \mathrm{H}$-atoms cm $\mathrm{cm}^{-2}$, which corresponds to the galactic dust layer. Forcing the fits to this value, the ROSAT and XMMNewton fits yield similar blackbody temperatures and a bolometric soft X-ray flux of $F_{\mathrm{X}} \simeq 1.5 \times 10^{-11} \mathrm{erg} \mathrm{cm}^{-2} \mathrm{~s}^{-1}$.

\section{Optical spectroscopy and spectropolarimetry}

\subsection{High-state spectra}

In Fig. 3, we show the mean low-resolution spectra taken on 13-17 December 1993 and 14 November 1995, when HY Eri was in high states. They are characterized by a blue continuum, strong Balmer, He I, and He II emission lines, the Balmer jump in emission, and weak broad cyclotron lines at the red end. Phase-resolved radial velocities were measured from mediumresolution blue spectra taken on 15 November 1995 (not shown).

\footnotetext{
6 http://vizier.u-strasbg.fr/viz-bin/VizieR?-source= $\mathrm{J} / \mathrm{A}+\mathrm{A} / 594 / \mathrm{A} 116$
}
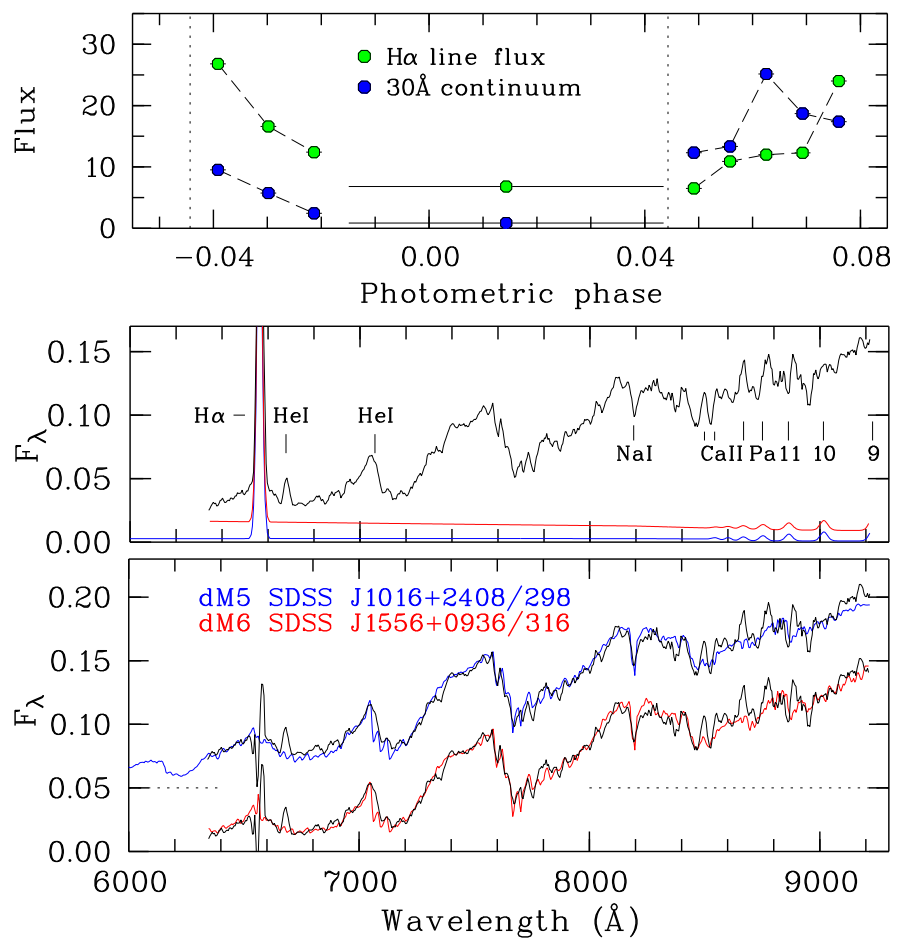

Fig. 4. Top: eclipse light curve of the $\mathrm{H} \alpha$ emission line flux in $\mathrm{erg} \mathrm{cm} \mathrm{cm}^{-2} \mathrm{~s}^{-1}$ and of the underlying continuum flux observed on 20 November 2000. Center: eclipse spectrum (black curve) thermal hydrogen spectrum adjusted to fit the $\mathrm{H} \alpha$ line flux (blue or red curve, see text). The ordinate is in units of $10^{-16} \mathrm{erg} \mathrm{cm}^{-2} \mathrm{~s}^{-1} \AA^{-1}$. Bottom: difference spectra on the same color coding fitted by a dM6 star (red) and a dM5 star (blue, shifted upward by 0.05 units).

The Balmer and HeII emission lines were single peaked with asymmetric bases, extending to beyond $\pm 1000 \mathrm{~km} \mathrm{~s}^{-1}$. We measured the mean central wavelengths of the broad components and the positions of the line peaks of $\mathrm{H} \beta, \mathrm{H} \gamma$, and HeII $\lambda 4686$. The former has a velocity amplitude of $520 \pm 24 \mathrm{~km} \mathrm{~s}^{-1}$ and reaches maximum positive radial velocity at $\phi=0.91 \pm 0.02$. This phasing is consistent with the plasma motion in the magnetically guided stream that leads to pole 1 and away from the observer at an azimuth of $\psi \sim 35^{\circ}$, measured from the line connecting the two stars. The line peak displays a small radial velocity with zero crossing near $\phi=0.80$. This component could arise from the ballistic accretion stream near $L_{1}$.

\subsection{Spectrum of the secondary star in eclipse}

On 20 November 2000, we acquired a spectrum of the secondary star during the WD eclipse, using the ESO VLT UT1 with FORS1 and grism G300I (Table 3). The 600 s exposure was preceded by three and followed by five $30 \mathrm{~s}$ exposures. The run started shortly after the ingress of the accretion spot into eclipse and extended until after its egress. The dotted vertical lines in (Fig. 4, top panel) indicate the duration of the eclipse. The $600 \mathrm{~s}$ exposure covered the phase interval $\phi=-0.0149$ to 0.0435 , beginning after the stream component subsided and ending just before the spot starts to reappear at $\phi=0.0438$. The figure shows the light curves of the $\mathrm{H} \alpha$ line flux and of the continuum near $\mathrm{H} \alpha$ integrated over $30 \AA$. The $\mathrm{H} \alpha$ emission stays finite in the eclipse.

The center panel of Fig. 4 shows the eclipse spectrum dereddened with the galactic extinction $A_{V}=0.172$ (Schlegel et al. 1998), where we have assumed that HY Eri is located outside 
the principal dust layer of the galactic disk (Jones et al. 2011). The secondary has a dereddened AB magnitude $i=20.99$ with an estimated systematic error of $0.10 \mathrm{mag}$ from the absolute flux calibration of the spectrophotometry. The spectrum shows the $\mathrm{TiO}$ features characteristic of a late $\mathrm{dM}$ star and a strong $\mathrm{H} \alpha$ emission line with a line flux of $6.8 \times 10^{-16} \mathrm{erg} \mathrm{cm}^{-2} \mathrm{~s}^{-1}$. The line is centered approximately at the rest wavelength and has a velocity dispersion of $\sim 1000 \mathrm{~km} \mathrm{~s}^{-1}$. It may arise from a tenuous uneclipsed section of the accretion stream. Regardless of its origin, the line emission will be accompanied by a thermal continuum that we need to define and subtract before a spectral type can be assigned to the secondary star. To this end, we calculated spectra of an isothermal hydrogen plasma of finite optical depth, added line broadening, and normalized the spectra to fit the observed $\mathrm{H} \alpha$ line flux. The free parameters of the model are the electron temperature $T_{\mathrm{e}}$, the pressure $P$, and the geometrical thickness $x$ of the emitting plasma. In the center panel of Fig. 4, we show two examples that bracket the permitted range of the flux of the sought-after continuum. The blue spectrum for $T_{\mathrm{e}}=10000 \mathrm{~K}, P=10$ dyne $\mathrm{cm}^{-2}, x=10^{8} \mathrm{~cm}$ features a low thermal continuum and the red curve for $T_{\mathrm{e}}=20000 \mathrm{~K}$, $P=200$ dyne $\mathrm{cm}^{-2}, x=10^{8} \mathrm{~cm}$ a high one. The bottom panel of Fig. 4 shows the observed spectrum with either one of the model spectra subtracted. Employing a set of dereddened spectra of SDSS dM stars, we find that the observed spectrum corrected with the low thermal continuum is best fitted with the dM5 star SDSS J101639.10+240814.2 adjusted by a factor of 287 (rms spectral flux deviation 0.0055 in the ordinate unit of Fig. 4, bottom panel). The corresponding spectrum for the high thermal continuum is equally well fitted with the dM6 star SDSS J155653.99+093656.5 adjusted by a factor of 289 (rms deviation 0.0053). The two cases tap the full range of thermal continua permitted by the eclipse spectrum and spectral types outside the dM5-6 slot quickly fail to provide an acceptable fit. For the dM5 case, the dereddened spectrum of the secondary corrected for the small thermal contribution has $i=21.03$ and colors $r-i=1.73$ and $i-z=0.95$. For the dM6 case with its larger thermal component, we find $i=21.22$ with $r-i=2.01$ and $i-z=1.10^{7}$. The angular radius $R_{2} / d$ of the secondary star and its brightness are related by the surface brightness

$S=m+5 \log \left[\left(R_{2} / d\right)\left(10 \mathrm{pc} / R_{\odot}\right)\right]$,

where $m$ is the magnitude of the star in the selected band, $R_{2}$ its radius, and $d$ its distance. We calibrated the surface brightness $S_{i}$ in the $i$-band as a function of color, using the extensive data set of Mann et al. (2015) that combines Sloan griz photometry and stellar parameters. The desired relations are

$$
\begin{array}{lrr}
S_{i}=5.34+1.554(r-i) & \text { with } & \sigma\left(S_{i}\right)=0.086 \\
S_{i}=5.33+2.829(i-z) & \text { with } & \sigma\left(S_{i}\right)=0.072
\end{array}
$$

valid for $r-i=1.0-2.6$ and $i-z=0.6-1.3$, respectively. The quoted standard deviations describe the average spread of $S_{i}$ around the fit. With the colors of the secondary star quoted above, we obtained mean values from Eqs. (4) and (5) of $S_{i}(\mathrm{dM} 5)=8.02$ and $S_{i}(\mathrm{dM} 6)=8.44$. From Eq. (3), the distance

\footnotetext{
7 We folded the SDSS spectrum over the Sloan $i$ filter curve for airmass zero, obtaining $i=14.89$ for SDSS J101639+240814 and $i=15.07$ for SDSS J155653+093656. Adding the distance moduli yields the quoted magnitudes. The $i$-band magnitudes of the SDSS spectra represent the appropriate reference for the present purpose, although they differ by +0.05 and -0.05 , respectively, from the DR 15 photometry. The quoted colors $r-i$ and $i-z$ are those of the SDSS photometry.
}

$d$ in pc is given by

$\log d=\left(i-S_{i}\right) / 5+1+\log \left(f_{\text {back }} R_{2} / R_{\odot}\right)$

where $i$ is the magnitude of the continuum-corrected eclipse spectrum, $S_{i}$ the respective surface brightness, $R_{2}$ the volume equivalent radius of the Roche lobe, and $f_{\text {back }} \simeq 0.961$ the reduction factor for the backside view of the lobe ${ }^{8}$. The dM5-dM6 differences in $S_{i}$ and in the $i$-band magnitude compensate in part, leading to similar distances with a ratio $d_{\mathrm{dM} 5} / d_{\mathrm{dM} 6}=1.11$. We employ Eq. (6) in Sect. 7.5, using the radii of the secondary star derived from our dynamic models of HY Eri. The error in the mean dM5-dM6 distance includes the \pm 0.10 mag uncertainty in the flux calibration, the \pm 0.08 mag scatter in $S_{i}$, and half the difference of the dM5 and dM6 distances. Added quadratically, the error in $d$ is $\pm 8.1 \%$ plus any error that arises from $R_{2}$.

We estimated the effective temperature of the secondary star from the color dependence of $T_{\text {eff }}$ in the data of Mann et al. (2015), which yields $T_{\text {eff }} \simeq 3070 \mathrm{~K}$ and $2900 \mathrm{~K}$ for the dM5 and the dM6 case, respectively. With the best-fit system parameters of Sect. 7.5, the luminosity of the secondary star becomes 2.6-2.0 $\times 10^{31} \mathrm{erg} \mathrm{s}^{-1}$ for a spectral type range of dM5-6.

\section{Circular spectropolarimetry}

We studied the magnetic field of the WD in HY Eri by phaseresolved circular spectropolarimetry performed on 31 December 2008 , when the system was accreting at a low level. A total of 40 sets of ordinary and extraordinary spectra were obtained with the ESO VLT UT2 equipped with FORS1 and GrismG300V, covering two consecutive orbital periods (Table 3 ). The pipeline reduction combines two spectra each for two positions of the $\lambda / 4$ plate in order to account for possible cross-talk from linear polarization. This procedure yielded set 1 of 20 calibrated intensity and circular polarization spectra, each covering a phase interval $\Delta \phi=0.10$. Spectral features faithfully repeated in both orbits and we phasefolded the intensity and polarization spectra for further analysis. In case of the intensity spectra, the phase resolution can be improved to $\Delta \phi=0.05$ by adding the individual ordinary and extraordinary spectra to form set 2 of 40 provisionally calibrated intensity spectra. Set 1 was employed for a quantitative analysis of the spectral flux, set 2 for tracing the motion of the cyclotron line peaks and measuring the $\mathrm{H} \alpha$ radial velocities. Both were corrected for the secondary star, using the dM5 representation of the eclipse spectrum of Fig. 4 extrapolated into the blue spectral region and set 1 was dereddened with $A_{V}=0.172$ (Schlegel et al. 1998). $\mathrm{H} \alpha$ emission is much weaker than in the 2000 eclipse spectroscopy of Sect. 5.2 and we do not correct for the probably tiny contribution of the associated thermal continuum. Grayscale representations of the corrected phasefolded set- 1 intensity and circular polarization spectra are shown in Fig. 5, repeated twice for visual continuity. Rows \#1, \#11, and \#21 represent the eclipse. The orbital mean spectrum outside eclipse (rows \#3-10, $\phi=0.14-0.91$ ) is shown in the left panel of Fig. 6 (red curve); an appropriate model spectrum of the magnetic WD is added for comparison (black curve). The two blue circles are the mean out-of-eclipse $g$ and $r$-band fluxes of our 2017 and 2018 photometry, corrected for the secondary. They show that the 2008, 2017, and 2018 observations were performed in similar states of low accretion.

\footnotetext{
8 The cross section of the secondary as seen along the line connecting the two stars is taken as elliptical with axes $y_{4}$ and $z_{6}$, yielding $\left.f_{\text {back }}=\sqrt{(} y_{4} z_{6}\right) /\left(r^{*}\right)_{2} \simeq 0.961$, in Kopal (1959) notation, with $\left(r^{*}\right)_{2}$ the equivalent volume filling radius of the Roche lobe.
} 

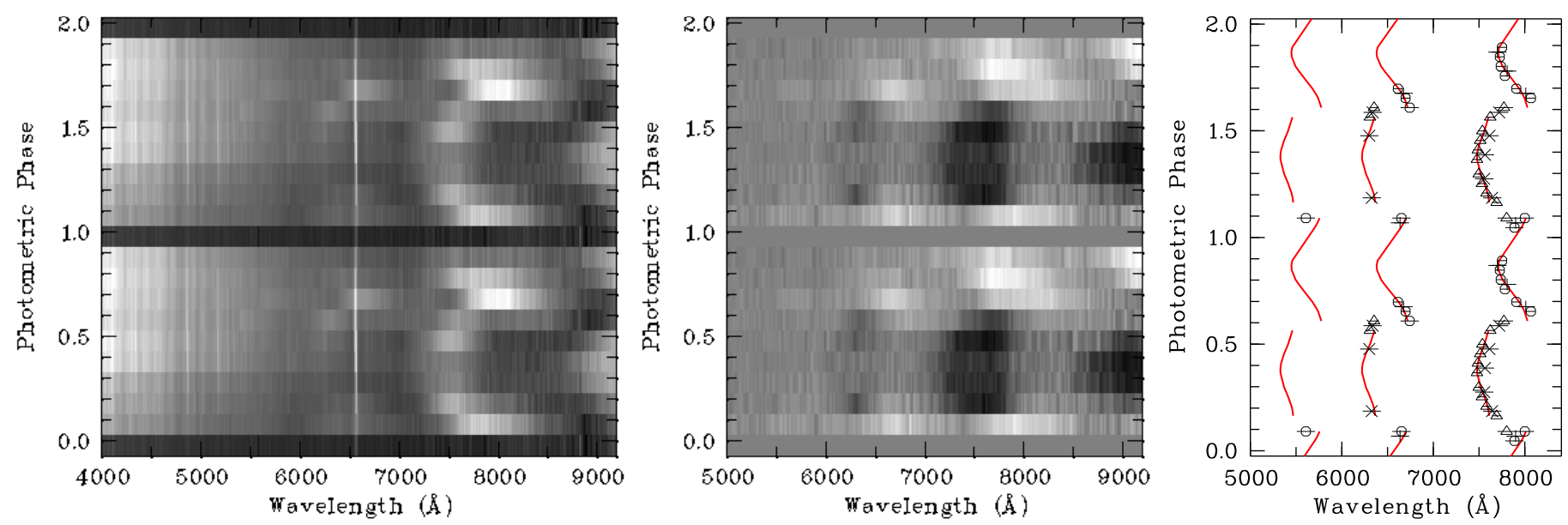

Fig. 5. Left: intensity spectra of the 2008 spectropolarimetry with the contribution of the secondary star subtracted. The mean spectra of two orbits are shown twice for visual continuity. Two systems of cyclotron lines are visible that originate near two poles of similar field strength. Center: circular polarization spectra with white indicating positive and black negative polarization. Right: maxima of the cyclotron lines of both poles (circles, triangles), extrema of the circular polarization (plus signs, crosses), and best-fit line positions as functions of orbital phase (red curves).
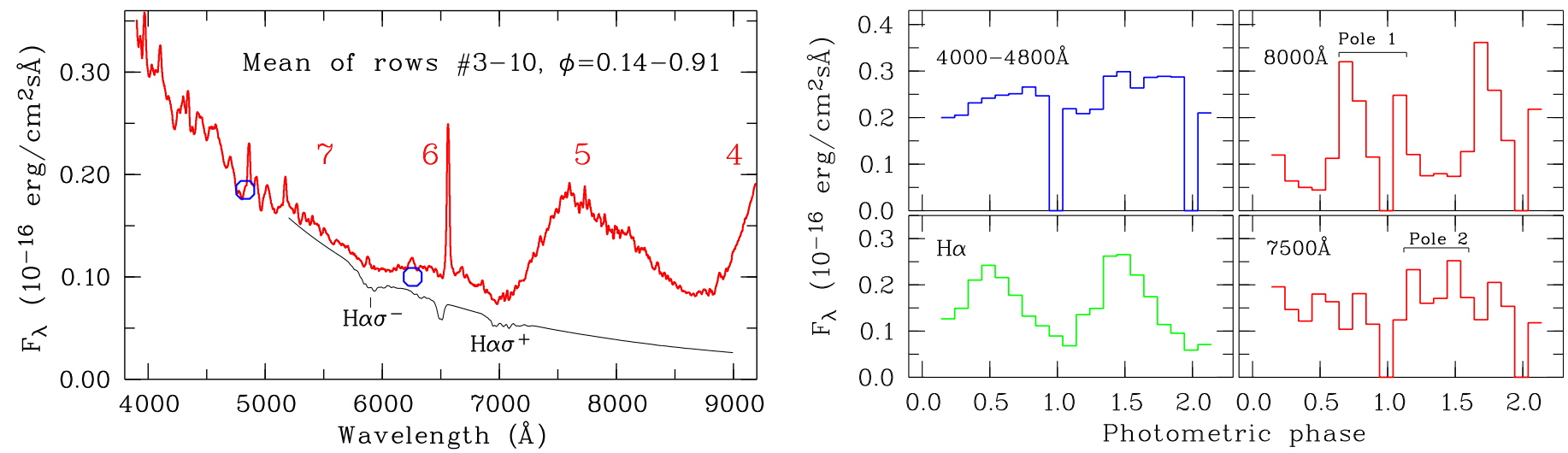

Fig. 6. Left: mean bright-phase dereddened intensity spectrum of the 2008 spectropolarimetry with the contribution of the secondary star subtracted (red curve). For comparison, the appropriate model spectrum of a magnetic WD is shown (black curve). The mean fluxes of the November 2017 and February $2018 \mathrm{gr}$ photometry are included as the two open blue circles. The red numbers indicate the cyclotron harmonics. Right: light curves for selected wavelength intervals derived from spectral set 1 before phasefolding, but after subtraction of the secondary star. The individual panels show the quasi-B band flux, the cyclotron beaming of the pole- 1 and pole- 2 emissions, and the $\mathrm{H} \alpha$ line flux (in arbitrary units).

The right panels of Fig. 6 show the light curves for the blue and red wavelength intervals extracted from the set 1 spectra. The red ones (7400-7600 $\AA$ and 7900-8100 $\AA$ ) are shaped by the beamed optically thin cyclotron emission of poles 1 and 2 . The blue one (4000-4800 ̊) represents the sum of the photospheric and spot emissions of the WD. Disentangling the two components proved infeasible. The $\mathrm{H} \alpha$ line emission is discussed in Sect. 7.3.

\subsection{Cyclotron spectroscopy}

The cyclotron lines of pole 1 are visible from $\phi=0.6-1.1$ with positive circular polarization and those of pole 2 in the remainder of the orbit with negative circular polarization. Overlaps between the two line systems occur in rows \#3 $(\phi=0.14-0.23)$ and and \#7 $(\phi=0.54-0.63)$, where the spectra show the signatures of both poles. We extracted the cyclotron lines from the individual intensity spectra by removing the underlying WD continuum. The cyclotron line profiles were then subjected to least squares fits using the theory of Chanmugam \& Dulk (1981) for an isothermal plasma. The free parameters of the model are the plasma temperature $k T$, the field strength $B$, the viewing angle $\theta$ against the field direction, the thickness parameter $\Lambda$ and a remnant optically thick continuum represented by a second-order polynomial (dashed lines in Fig. 7). The thickness parameter $\Lambda$ of cyclotron theory is related to the column density $x_{\mathrm{s}}$ of the cooling region by $\Lambda=4 \pi e x_{\mathrm{s}} / \mu_{\mathrm{e}} m_{\mathrm{u}} B$, where $e$ is the elementary charge, $\mu_{\mathrm{e}}$ the number of electrons per nucleon in the plasma, $m_{\mathrm{u}}$ is the atomic mass unit, and $B$ the field strength.

Because of trade-off effects between $k T$ and $\log \Lambda$, reliable values of these two parameters can not be determined without additional information. We took recourse to the results of the two-fluid radiation-hydrodynamic cooling theory of Fischer \& Beuermann (2001) and explain our approach for the case of the pole-2 spectrum of row \#6 in the lower left panel of Fig. 7. Good fits to that spectrum were obtained along a narrow valley in the $\Lambda-k T$ plane that follows $\log \Lambda=6.64-4.73 \log (k T)$ and extends to parameters quite inappropriate for a post-shock region dominated by cyclotron cooling. Cooling theory provides a second relation between $\log \Lambda$ and $\log (k T)$ that runs nearly orthogonal to that of the line fits. For the case of the \#6 spectrum, it reads 

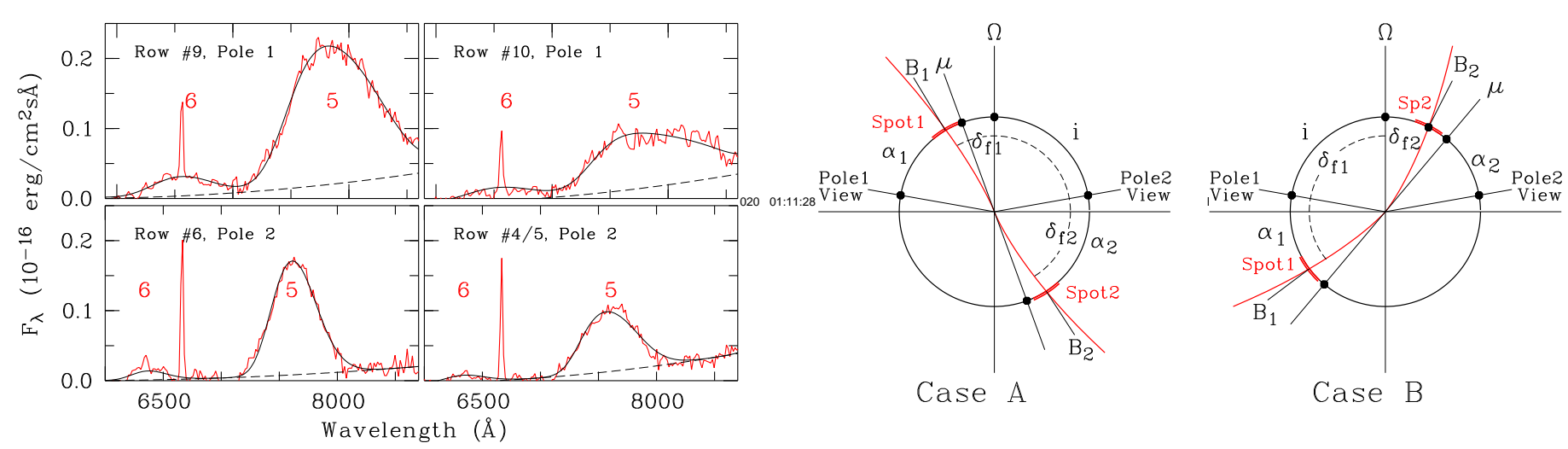

Fig. 7. Left: cyclotron spectra of poles 1 and 2 (red curves) least-squares fitted with constant-temperature models for the plasma parameters listed in Table 6 (black curves). Right panels: magnetic geometry of the WD for case A and case B accretion (see text). The secondary star is located to the left. Angles are measured between the dots on the circumference. Dipolar field lines are included as red curves.

Table 6. Physical parameters of spots 1 and 2 derived from least squares fits of the model of an isothermal plasma to the cyclotron line profiles.

\begin{tabular}{ccccccccc}
\hline \hline $\begin{array}{c}\text { Pole Row } \\
\#\end{array}$ & $\phi$ & $\begin{array}{c}\text { Harmonics } \\
\text { fitted }\end{array}$ & $\begin{array}{c}B_{\mathrm{sp}} \\
(\mathrm{MG})\end{array}$ & $\begin{array}{c}k T \\
(\mathrm{keV})\end{array}$ & $\begin{array}{c}\langle\theta\rangle \\
\left({ }^{\circ}\right)\end{array}$ & $\log \Lambda$ & $\begin{array}{c}\dot{m} \\
\left(\mathrm{~g} \mathrm{~cm}^{-2} \mathrm{~s}\right)\end{array}$ \\
\hline 1 & 2 & 0.07 & $(4) 56$ & 27.50 & 2.25 & 63 & 5.84 & 0.0095 \\
1 & 10 & 0.87 & $(4) 56$ & 27.37 & 2.20 & 49 & 5.81 & 0.0090 \\
1 & 9 & 0.78 & $(4) 56$ & 27.51 & 2.25 & 59 & 5.84 & 0.0102 \\
2 & 6 & 0.48 & $(4) 56$ & 28.83 & 1.70 & 68 & 5.55 & 0.0048 \\
2 & $4 / 5$ & 0.33 & $(4) 56$ & 28.88 & 1.65 & 62 & 5.50 & 0.0040 \\
\hline
\end{tabular}

$\log \Lambda=4.97+2.50 \log (k T)^{9}$. The intersection of the two relations defines the most probable values of $\log \Lambda$ and $k T$, yielding $k T=1.70 \mathrm{keV}$ and $\log \Lambda=5.55$. Table 6 lists the corresponding fits to all spectra that can uniquely be assigned to either pole 1 or pole 2 . The emerging picture is that of two emission regions with similar field strengths $B_{\mathrm{sp}} \simeq 27.5 \mathrm{MG}$ and $28.8 \mathrm{MG}$, thickness parameters $\log \Lambda \simeq 5.8$ and 5.5 , and temperatures of $k T \simeq 2.2 \mathrm{keV}$ and $1.7 \mathrm{keV}$ for poles 1 and 2 , respectively. The third parameter derived from the cyclotron fits is the mean viewing angle $\langle\theta\rangle$ between the line of sight and the direction of the accreting field line averaged over the spot. Closest approach to the field line occurs for pole 1 at $\phi \simeq 0.87$ in row \#10 and for pole 2 at $\phi \simeq 0.33$ in rows \#4 and \#5 with minimum viewing angles of $\left\langle\theta_{\min , 1}\right\rangle \simeq 49^{\circ}$ and $\left\langle\theta_{\min , 2}\right\rangle \simeq 62^{\circ}$ for spots 1 and 2 , respectively. Since the cyclotron lines widen and weaken rapidly with decreasing $\theta$, the quoted angles may somewhat overestimate the true mean values.

Table 6 also lists the mass flow densities $\dot{m}$ that are delivered also by two-fluid cooling theory. They fall far below the $\sim 1 \mathrm{~g} \mathrm{~cm}^{-2} \mathrm{~s}^{-1}$ of a bremsstrahlung-dominated emission region and are only about an order of magnitude away from the transition to the non-hydrodynamic regime of the bombardment solution (Woelk \& Beuermann 1992; Fischer \& Beuermann 2001).

\footnotetext{
9 The second $\Lambda-k T$ relation is based on equations for the post-shock plasma temperature $k T$ and the column density $x_{\mathrm{s}}$ of the post-shock cooling flow presented in Figs. 5 and 6 and Eqs. (19) and (20) of Fischer \& Beuermann (2001). Elimination of the variable $\dot{m} B_{7}^{-2.6}$, with $\dot{m}$ the mass-flow density in $\mathrm{g} \mathrm{cm}^{-2} \mathrm{~s}^{-1}$ and $B_{7}$ the field strength in units of $10^{7} \mathrm{G}$, yields the desired relation between $k T$ and $\Lambda\left(x_{\mathrm{s}}\right)$, which is well fitted by a power law valid for $k T$ up to about $3 \mathrm{keV}$. For the present purpose, the relations in question were re-calculated for a plasma of solar composition and a WD mass $M_{1}=0.4 M_{\odot}$ as suggested by our dynamic models in Sect. 7.5.
}

Table 7. Geometry of the accreting field lines derived from least squares fits to the orbital motion of the cyclotron lines (Fig. 5).

\begin{tabular}{lcccccc}
\hline \hline Pole & $\begin{array}{c}B_{\mathrm{sp}} \\
(\mathrm{MG})\end{array}$ & $\begin{array}{c}\delta_{\mathrm{f}} \\
\left({ }^{\circ}\right)\end{array}$ & $\begin{array}{c}\psi_{\mathrm{f}} \\
\left({ }^{\circ}\right)\end{array}$ & $\begin{array}{c}\theta_{\min } \\
\left({ }^{\circ}\right)\end{array}$ & $\begin{array}{c}\angle\left(\boldsymbol{B}_{\mathrm{sp}, 1}, \boldsymbol{B}_{\mathrm{sp}, 2}\right) \\
\left({ }^{\circ}\right)\end{array}$ & $\Delta(\phi)$ \\
\hline $1 \mathrm{~N}$ & 27.2 & 36 & 49 & 45 & & \\
$2 \mathrm{~S}$ & 28.6 & 136 & 224 & 56 & 170.9 & 0.487 \\
$1 \mathrm{~S}$ & 27.3 & 126 & 57 & 46 & & \\
$2 \mathrm{~N}$ & 28.4 & 28 & 227 & 52 & 153.2 & 0.472 \\
\hline
\end{tabular}

Complementary information on $\theta$ is obtained from the orbital motion of the cyclotron line peaks and of the circular polarization extrema. The peak wavelengths measured from the spectra of sets 2 and 1, respectively, are shown in the right panel of Fig. 5. Near $\phi=0.1$ and 0.6 , both line systems overlap and are difficult to disentangle. As in Paper I, the motion of the cyclotron lines was modeled, using a parameterized form of the frequencies of optically thin harmonics in units of the cyclotron frequency as functions of $k T$ and $\theta$. We defined the field vectors in the two spots as $\boldsymbol{B}_{\mathrm{sp}, 1}$ and $\boldsymbol{B}_{\mathrm{sp}, 2}$ and obtained field strengths and directions by least-squares fitting the phase-dependent motion of the line peaks of the fifth and sixth harmonics (red curves in Fig. 5). As input we used the plasma temperatures of Table 6 and an inclination of $i=80^{\circ}$ from Table 9. The results are presented in Table 7, where we list the field strengths $B$, the azimuth angles $\psi_{\mathrm{f}}$, and the colatitudes $\delta_{\mathrm{f}}$ of the accreting field lines. The results are quoted for two accretion geometries with pole 1 either in the "southern" hemisphere below the orbital plane (1S) or in the "northern" one above it $(1 \mathrm{~N})$. Note that the fit does not provide information on the location of the spots and the orientation of the magnetic axis. The angle between the field vectors $\boldsymbol{B}_{\mathrm{sp}, 1}$ and $\boldsymbol{B}_{\mathrm{sp}, 1}$ is not far from $180^{\circ}$, at least in the $1 \mathrm{~N}-2 \mathrm{~S}$ case. Combined with the fact, that both spots display circular polarization of opposite sign, the data suggest a field structure that is dominated by a dipole and possibly octupole rather than a quadrupole.

\subsection{Zeeman spectroscopy}

The blue continuum in the left panel of Fig. 5 represents the photospheric emission of the WD including a strong spot component. To facilitate the Zeeman analysis, we transformed the set-1 spectra to the rest system of the WD, employing the preferred dynamical model of Sect. 7.5. There is little orbital variation in the Zeeman lines except near the transitions between 

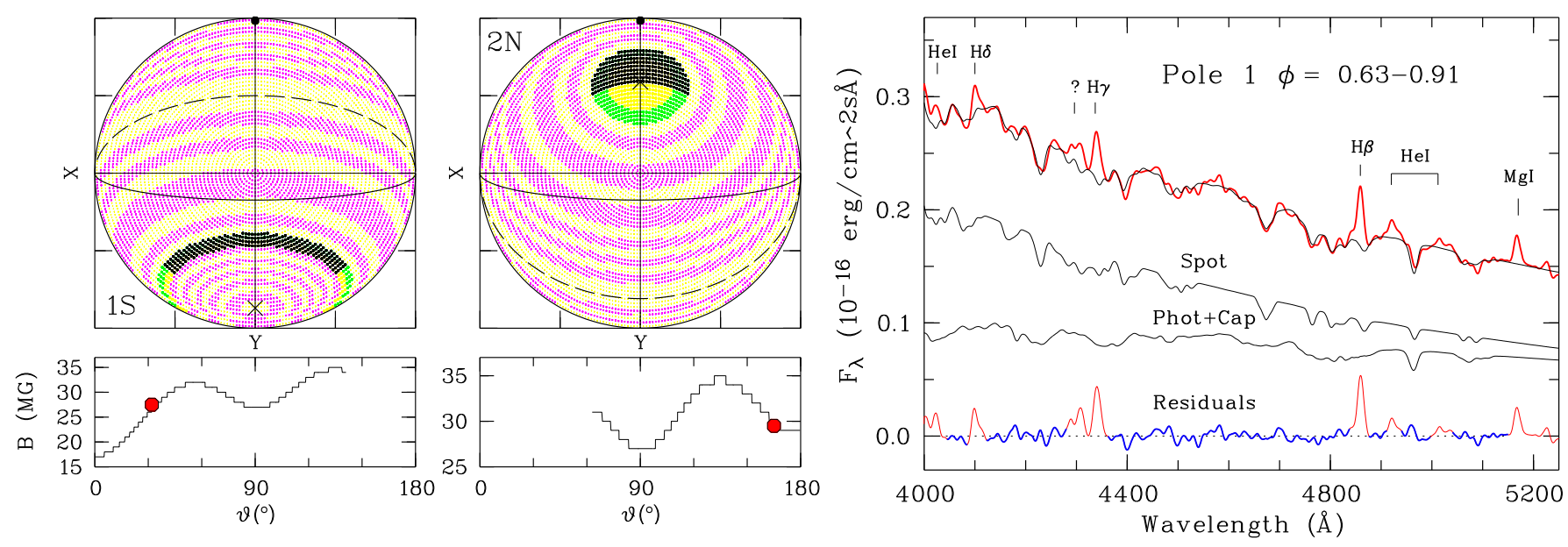

Fig. 8. Left panels: magnetic-field distributions of the WD for the case B multipole model of Table 8 and the phases of best visibility of spots $1 \mathrm{~S}$ and $2 \mathrm{~N}$. The footpoints of the rotational and the magnetic axes are indicated by the black dot and the cross $(\times)$, respectively. The magnetic equator and the orbital plane are marked by the dashed and the solid black line, respectively. The individual projected-area elements are marked by tiny dots, selecting or suppressing every second integer field strength. The spot field is marked in green and the viewing-angle selected spot is emphasized in black. The bottom panels show the model field strength vs. the magnetic colatitude $\vartheta$. The red dots mark the spot fields. Right panel: observed pole-1 spectrum (red curve) with the best-fit Zeeman spectrum for the adopted multipole model (black curves). The residuals of the fit are displayed at the bottom with the wavelength sections that are included in the fit highlighted in blue.

the visibility of poles 1 and 2 . We adopted the averages of rows $\# 8-10(\phi=0.63-0.91)$ or \#3-6 ( $\phi=0.14-0.52)$ as representative of the hemispheres that include pole 1 or 2 , respectively. The dominant field strengths are 27-28 MG in pole 1 and 29-30 MG in pole 2 . The $4000-5250 \AA$ section of the pole- 1 spectrum is shown in Fig. 8 (red curve). Unfortunately, the circular polarization spectra, which contain information on the direction of the magnetic field vector $\boldsymbol{B}$, are too noisy to be of any use, limiting our ability to distinguish between different magnetic field structures that fit the intensity spectra similarly well.

Our spectral synthesis program employs an improved set of the model Zeeman spectra calculated by Jordan (1992) and previously used by Euchner et al. (2002, 2005, 2006) and Beuermann et al. (2007). The present version includes the Balmer lines up to $\mathrm{H} \delta$ and consists of the $\log g=8$ intensity spectra for 16 effective temperatures from 8 to $100 \mathrm{kK}$, integer field strengths $B$ from 1 to $100 \mathrm{MG}$, and 17 viewing angles $\theta=0^{\circ}$ to $180^{\circ}$, uniformly distributed in $\cos \theta$. The model spectra were calculated for a Stark broadening factor $C=0.1$ (Jordan 1992). Interpolation in $T_{\text {eff }}$ and in $\theta$ is unproblematic, while interpolation in $B$ is impracticable. The spectra were smoothed to match the observed resolution of $10 \AA F W H M$. At this resolution, the 1-MG spacing is just about adequate and misfits stay small.

We considered a magnetic model that includes the zonal multipole components of degree $\ell=1-3$, that is the aligned dipole, quadrupole, and octupole, inclined by a common angle $\alpha$ against the line of sight. For the relative field strengths $r_{\text {dip }}=1-r_{\mathrm{oct}}, r_{\mathrm{qua}}$, and $r_{\text {oct }}$, the combined polar field strength is $B_{\mathrm{p}}=\left(1+r_{\text {qua }}\right) B_{0}$, with $B_{0}$ a scaling factor. We divided the visible disk of the WD into 6561 limb-darkened projected area elements, and collected them into $17 n$ field-strength and viewing-angle bins $(k, l), k=$ $1 \ldots n$ for the nearest integer field strength $B_{\mathrm{k}}$ and $l=1 \ldots 17$ for the nearest $\cos \theta_{1}$ value. The unreddened spectral flux at the Earth is

$f_{\lambda}\left(B_{0}, r_{\mathrm{oct}}, \alpha\right)=\left(R_{w \mathrm{~d}} / d\right)^{2} \sum_{k=1}^{\mathrm{n}} \sum_{l=1}^{17} a_{k, l} F_{\lambda}\left(B_{k}, T_{k, l}, \theta_{l}\right)$,

where $a_{k, l}$ is the integrated limb-darkened fractional projectedarea of bin $(k, l), F_{\lambda}\left(B_{k}, T_{k, l}, \theta_{l}\right)$ the data bank spectrum for that bin at the interpolated temperature $T_{k, l}$, and $\left(R_{w d} / d\right)^{2}$ the dilution factor, with $R_{w \mathrm{~d}}$ the WD radius and $d$ the distance. The best values of $B_{0}, r_{\text {qua }}, r_{\text {oct }}$, and $\alpha$ were determined in a grid search, the $T_{k, l}$ and the dilution factor by a formal least squares fit at each grid point. Obtaining a stable fit, requires a severely restricted number of independent temperatures. The spot emits about $2 / 3$ of the blue flux from $\sim 5 \%$ of the area, requiring a minimum of two temperatures, naturally identified as a high spot temperature $T_{\mathrm{sp}}$ and a low photospheric temperature $T_{\mathrm{ph}}$. Some fits benefit from a minor third component, such as a warm polar cap with $T_{\text {cap }}$. We extended the fit over the wavelength interval 4000-5200 $\AA$, excluding sections around the Balmer emission lines, some HeI lines, and an unidentified line complex around $4300 \AA$. A formal $\chi^{2}$ was calculated for 85 resolution elements of $10 \AA$ width, using relative flux errors of $1.8 \%$ for pole 1 and $2.4 \%$ for pole 2 , measured from the scatter among the set- 1 intensity spectra.

\subsection{Stark broadening}

An accepted theory of Stark broadening in the presence of a magnetic field does not exist. Jordan (1992) opted to equate the broadening of the individual Stark components of a Balmer line to the mean Stark shift of all components multiplied by a factor $C \simeq 0.1$ and Putney \& Jordan (1995) considered values of $C=0.1$ and 1.0 for stars with vastly different field strengths. It is necessary, therefore, to consider the appropriate level of the line broadening for a given application. To this end, we adopted an approximate post factum procedure that changes the line strengths, while avoiding recalculation of the data base. We expressed the line profiles in terms of an optical depth $\tau_{\lambda}$, setting $F_{\lambda}=F_{\lambda}^{\mathrm{c}} e^{-\tau_{\lambda}}$, with $F_{\lambda}^{\mathrm{c}}$ the continuum flux. We then replaced $F_{\lambda}$ in Eq. (7) by $F_{\lambda}^{\text {new }}=F_{\lambda}^{\mathrm{c}} e^{-\tau_{\lambda} \eta}$ and included $\eta$ as an additional free parameter in the grid search. For our best multipole model, we obtained $\chi^{2}=85.5$ at $\eta=1(C=0.1)$, the best fit with $\chi_{\min }^{2}=84.8$ was attained at $\eta=1.25$, and the $90 \%$ confidence level with $\chi_{\min }^{2}=87.5$ was reached for $\eta=0.80$ and 2.50 , with $\chi^{2}$ quickly rising at still lower and higher $\eta$. Hence, our fit favors line strengths somewhat larger than nominal $(C=0.10, \eta=1.0)$. 
A\&A 634, A91 (2020)

Table 8. Physical parameters derived from common Zeeman fits to the spectra of poles 1 and 2 for a quasi-dipole and the best multipole model.

\begin{tabular}{|c|c|c|c|c|c|c|c|c|c|c|c|c|c|c|c|c|c|c|c|c|}
\hline $\begin{array}{l}\text { (1) } \\
\text { Pole }\end{array}$ & $\begin{array}{l}\text { (2) } \\
r_{\text {gua }}\end{array}$ & $\begin{array}{l}\text { (3) } \\
r_{\text {oct }}\end{array}$ & $\begin{array}{l}(4) \\
B_{0}\end{array}$ & $\begin{array}{l}\text { (5) } \\
\alpha\end{array}$ & $\begin{array}{l}(6) \\
\delta_{\mu}\end{array}$ & $\begin{array}{l}(7) \\
\left\langle\delta_{\mathrm{f}}\right\rangle\end{array}$ & $\begin{array}{l}(8) \\
\left\langle\theta_{\mathrm{f}}\right\rangle\end{array}$ & $\begin{array}{c}(9) \\
\left\langle\vartheta_{\mathrm{sp}}\right\rangle\end{array}$ & $\begin{array}{l}(10) \\
\left\langle\beta_{\mathrm{sp}}\right\rangle\end{array}$ & $\begin{array}{l}(11) \\
\left\langle f_{\mathrm{sp}}\right\rangle\end{array}$ & $\begin{array}{l}(12) \\
B_{\mathrm{sp}}\end{array}$ & $\begin{array}{l}(13) \\
B_{\text {pole }}\end{array}$ & $\begin{array}{l}(14) \\
B_{\mathrm{dip}}\end{array}$ & $\begin{array}{l}(15) \\
B_{\text {qua }}\end{array}$ & $\begin{array}{l}(16) \\
B_{\mathrm{oct}}\end{array}$ & $\begin{array}{c}(17) \\
B_{\min }-B_{\max }\end{array}$ & $\begin{array}{l}(18) \\
T_{\text {phot }}\end{array}$ & $\begin{array}{c}(19) \\
R_{\mathrm{wd}} / d\end{array}$ & $\begin{array}{l}(20) \\
\chi^{2}\end{array}$ & $\begin{array}{l}\text { (21) } \\
\text { Note }\end{array}$ \\
\hline Pole & & $r_{\text {oct }}$ & $\begin{array}{c}B_{0} \\
(\mathrm{MG}) \\
\end{array}$ & $\begin{array}{l}\alpha \\
\left(^{\circ}\right) \\
\end{array}$ & $\begin{array}{l}\delta_{\mu} \\
\left({ }^{\circ}\right)\end{array}$ & $\begin{array}{l}\left\langle\delta_{\mathrm{f}}\right\rangle \\
\left({ }^{\circ}\right)\end{array}$ & $\begin{array}{c}\left\langle\theta_{\mathrm{f}}\right\rangle \\
\left(^{\circ}\right)\end{array}$ & $\begin{array}{c}\left\langle\vartheta_{\mathrm{sp}}\right\rangle \\
\left({ }^{\circ}\right)\end{array}$ & $\begin{array}{c}\left\langle\beta_{\mathrm{sp}}\right\rangle \\
\left({ }^{\circ}\right)\end{array}$ & $\left\langle f_{\mathrm{sp}}\right\rangle$ & $\begin{array}{c}B_{\mathrm{sp}} \\
(\mathrm{MG})\end{array}$ & $\begin{array}{l}B_{\text {pole }} \\
\text { (MG) }\end{array}$ & $\begin{array}{c}B_{\mathrm{dip}} \\
(\mathrm{MG})\end{array}$ & $\begin{array}{r}B_{\text {qua }} \\
(\mathrm{MG})\end{array}$ & $\begin{array}{l}B_{\text {oct }} \\
(\mathrm{MG})\end{array}$ & $\begin{array}{c}B_{\min }-B_{\max } \\
(\mathrm{MG})\end{array}$ & $\begin{array}{l}I_{\text {phot }} \\
(\mathrm{kK})\end{array}$ & $\begin{array}{c}K_{\mathrm{wd} /} / d \\
\left(10^{9} \mathrm{~cm} \mathrm{kpc}^{-1}\right)\end{array}$ & & Note \\
\hline \multicolumn{21}{|c|}{ (a) Quasi-dipole: } \\
\hline $1 \mathrm{~N}$ & -0.05 & 0.00 & 38.5 & 37 & 113 & 44 & 52 & 52 & 31 & 0.054 & $27-28$ & 36.6 & 38.5 & -1.9 & 0.0 & $19-37$ & $9.2(0.7)$ & $1.07(11)$ & 88.6 & (1) \\
\hline $2 \mathrm{~S}$ & 0.05 & 0.00 & 38.5 & -18 & 63 & 126 & 69 & 50 & 32 & 0.055 & $29-30$ & 40.4 & 38.5 & 1.9 & 0.0 & $19-40$ & $9.3(0.7)$ & $1.06(11)$ & 99.4 & (1) \\
\hline \multicolumn{21}{|c|}{ (b) Multipole: } \\
\hline $1 \mathrm{~S}$ & -0.25 & -0.77 & 22.8 & 61 & 141 & 113 & 40 & 34 & 1 & 0.041 & $27-28$ & 17.1 & 40.4 & -5.7 & -17.6 & $17-35$ & $9.2(0.8)$ & $1.15(12)$ & 84.6 & (2) \\
\hline $2 \mathrm{~N}$ & 0.25 & -0.77 & 22.8 & -41 & 39 & 37 & 45 & 11 & 0 & 0.044 & $29-30$ & 28.5 & 40.4 & 5.7 & -17.6 & $27-35$ & $9.2(0.8)$ & $1.14(12)$ & 90.0 & (3) \\
\hline
\end{tabular}

Notes. Columns (18) and (19) quote the nominal values of $T_{\mathrm{ph}}$ and $R_{\mathrm{wd}} / d$ from the Zeeman fit with the estimated systematic errors. (1) Unpassable access: field lines curve away from orbital plane. (2) Regular access from orbital plane. (3) Access over the rotational pole.

This result applies to our simultaneous multi-temperature fits to spectral flux and line strengths and may not be generally valid. We adopted $\eta=1.25$ for the present paper and obtained the systematic errors at the $90 \%$ confidence level for $\eta=0.8-2.5$. The large errors re-emphasize the need for an effort to calculate the Stark shifts of the individual Stark components in a magnetic field.

\subsection{Magnetic geometry of the accreting WD}

The right-hand panels of Fig. 7 show two selected magnetic WD geometries. For simplicity, both have rotational pole, magnetic pole, and the accretion spots on the same meridian (here the paper plane). The secondary star is located far to the left. The footpoints of the common magnetic axis are displaced from the respective viewing directions by $\alpha_{1}$ and $\alpha_{2}$, with $\alpha_{1}+\alpha_{2}=180^{\circ}-2 i$, where $i$ is the inclination and $\alpha$ is counted positive away from and negative toward the rotational pole. In case A, both spots are located between magnetic pole and viewing direction and can accrete from the nearby orbital plane. To reach spot 2 , the plasma must travel halfway around the WD before it attaches to a near-polar field line. In case B, spot 1 can accrete from the nearby orbital plane. Spot 2, however, is located between magnetic and rotational pole and the field line leads over the rotational pole in the general direction of the secondary star. Although energetically unfavorable, this non-standard path may be active and it is not clear whether the trip over the pole or the travel around the WD should be dismissed as the less likely way to feed spot 2 .

In perusing parameter space, we found that all good fits require field strengths larger than the spot field $B_{\mathrm{sp}}$ and are rather insensitive to a lack of small field strengths. We started from a pure dipole model that fits the pole 1 and pole 2 spectra with $B_{0}=37.5$ and $40.5 \mathrm{MG}$, respectively. Adding a small quadrupole component, leads to a common $B_{0}=38.5 \mathrm{MG}$. The parameters of this quasi-dipole fit are listed in Table 8. As expected for an inclination of $80^{\circ}$ (Sect. 7.5 and Table 9), $\alpha_{1}+\alpha_{2} \simeq 20^{\circ}$, confirming the presence of a common magnetic axis for the separately performed fits. The colatitudes $\delta_{\mathrm{f}}$ of the accreting field lines agree reasonably well with those of the $1 \mathrm{~S}-2 \mathrm{~N}$ geometry in Table 6 , considering the uncertainties of about $5^{\circ}$. Superficially, the fit seems close to perfect were it not for the disturbing fact that the geometry probably prevents accretion in both spots. The ribbon-like spots are offset from the respective magnetic pole by $\vartheta_{\text {sp }} \sim 50^{\circ}$, the field lines in the spots reach out to only $1.7 R_{\mathrm{wd}}$, and both field lines curve away from the orbital plane. Hence, the quasi-dipole model provides no convincing accretion geometry. Increasing the quadrupole component provides no remedy. For $r_{\text {qua }}$ up to \pm 0.40 , none of the seemingly good fits matches the requirements set up by the cyclotron fits. The same holds for moderate octupole components $r_{\text {oct }}$ up to \pm 0.40 , some of which predict "spots" in the form of near-equatorial ribbons connected by tightly closed field lines.

The situation changes fundamentally for larger octupole components with $r_{\text {oct }} \lesssim-0.45$. With decreasing $r_{\text {oct }}$, the bestfit values of $B_{0}$ in the primary minima of poles 1 and 2 converge and coincide for $r_{\text {oct }}=-0.77$ and $r_{\text {qua }}=\mp 0.25$, respectively. Table 8 lists the fit parameters. As required, $\alpha_{1}+\alpha_{2} \simeq 20^{\circ}$ and the spot-averaged colatitudes $\left\langle\delta_{\mathrm{f}}\right\rangle$ and viewing angles $\left\langle\theta_{\mathrm{f}}\right\rangle$ agree reasonably well with the $1 \mathrm{~S}-2 \mathrm{~N}$ cyclotron results of Table 6 . Both spots are located closer to the magnetic poles than in the quasidipole case and the field lines are close to radial with inclinations $\left\langle\beta_{\mathrm{f}}\right\rangle \simeq 0$, indicating that the field lines reach far out. The multipole model represents a convincing solution, provided the case $B$ path to spot 2 is active. In passing, we note that enforcing case A accretion at spot 2 by increasing its colatitude fails because a corresponding $\chi^{2}$ minimum does not exist. Switching hemispheres, the $1 \mathrm{~N}$ geometry is a mirror image of $1 \mathrm{~S}$, but a $\chi^{2}$ minimum at the parameters expected for $2 \mathrm{~S}$ does not exist either. The general caveat holds that a multipole model with tesseral harmonics may provide a different answer.

The two left panels of Fig. 8 show the magnetic field distributions of the WD for the multipole model of Table 8 at the phases of the best visibility of spots $1 \mathrm{~S}$ and $2 \mathrm{~N}$. The yellow and green bands indicate the spot field strengths of 27 and $28 \mathrm{MG}$ for pole 1 and 29 and $30 \mathrm{MG}$ for pole 2 and the black portions the viewingangle selected spots. Although both aspects belong to the same field model, the spot geometries differ significantly. So do the full ranges of the field strengths over the visible face of the WD (bottom panels, see also Col. 17 of Table 8). The right panel of Fig. 8 shows the pole- 1 spectrum (red curve) and the Zeeman fit (superposed black curve), which faithfully reproduces most Zeeman lines in the spectral regions that are free of atomic emission lines. The contributions by the spot and the photosphere+cap are also shown individually. The Zeeman lines are prominent in the spot component because of the small spread in field strength, but are washed out in the photospheric component. The fit to the pole- 2 spectrum (not shown) excels at $\lambda>4500 \AA$, but is inferior at shorter wavelengths, possibly because it is composed of only nine independent magnetic spectra (Table 8, Col. 17).

\subsection{WD parameters and their errors}

The multipole Zeeman fit to the observed pole 1 and pole 2 spectra yielded temperatures for the photosphere, cap, and spot of $T_{\mathrm{ph}}=9.2 \pm 0.7 \mathrm{kK}$ (Table 8$)$ and $T_{\text {cap }}=14.2 \pm 1.0 \mathrm{kK}$ and $T_{\mathrm{sp}}=78 \pm 8 \mathrm{kK}$ (Table 9). In most fits, the flux contributed by the cap is a minor entity. The corresponding angular radius of the WD is $R_{\mathrm{wd}} / d=(1.15 \pm 0.12) \times 10^{9} \mathrm{~cm} \mathrm{kpc}^{-1}$. All quoted 
K. Beuermann et al.: Neglected X-ray discovered polars. II.

Table 9. System parameters of HY Eri for models with main sequence and bloated secondary stars, derived with the radial velocity amplitude of the $\mathrm{Mg}$ I line and the irradiation model BT08.

\begin{tabular}{|c|c|c|c|c|c|c|c|c|c|c|c|c|c|c|c|c|c|}
\hline (1) & $(2)$ & (3) & (4) & (5) & $(6)$ & (7) & $(8)$ & (9) & $(10)$ & $(11)$ & $(12)$ & (13) & (14) & (15) & (16) & (17) & (18) \\
\hline Row & $\begin{array}{c}K_{2}^{\prime} \\
\left(\mathrm{km} \mathrm{s}^{-1}\right)\end{array}$ & $f_{3}$ & $\begin{array}{c}i \\
\left(^{\circ}\right) \\
\end{array}$ & $q$ & $\begin{array}{c}M_{2} \\
\left(M_{\odot}\right)\end{array}$ & $\begin{array}{c}R_{2} \\
\left(R_{\odot}\right) \\
\end{array}$ & $K_{2} / K_{2}^{\prime}$ & $\begin{array}{r}K_{\mathrm{L} 1} \\
(\mathrm{~km}\end{array}$ & $\begin{array}{l}K_{\text {term }} \\
\left.\mathrm{s}^{-1}\right)\end{array}$ & $\begin{array}{c}M_{1} \\
\left(M_{\odot}\right)\end{array}$ & $\begin{array}{l}R_{1, \mathrm{He}} \\
\quad\left(10^{9}\right.\end{array}$ & $\begin{array}{l}R_{1, \mathrm{CO}} \\
\mathrm{cm})\end{array}$ & $\begin{array}{c}d \\
(\mathrm{pc})\end{array}$ & $\begin{array}{c}R_{1, \mathrm{sp}} \\
\left(10^{9} \mathrm{~cm}\right)\end{array}$ & $R_{1} / R_{1, \mathrm{sp}}$ & $\begin{array}{c}R_{1} / d \\
\left(10^{9} \mathrm{~cm} \mathrm{kpc}^{-1}\right)\end{array}$ & $\begin{array}{c}T_{\mathrm{ph}} \\
(\mathrm{kK}) \\
\end{array}$ \\
\hline \multicolumn{18}{|c|}{ (a) Main sequence models: } \\
\hline 1 & 129 & 1.000 & 79.1 & 0.656 & 0.308 & 0.320 & 1.83 & 57 & 186 & 0.469 & 1.116 & & $1167 \pm 95$ & $1.343 \pm 0.189$ & 0.83 & 0.956 & 10.19 \\
\hline 2 & 139 & 1.000 & 79.8 & 0.615 & 0.305 & 0.318 & 1.76 & 67 & 196 & 0.497 & 1.065 & & $1160 \pm 94$ & $1.334 \pm 0.188$ & 0.80 & 0.918 & 10.42 \\
\hline 3 & 149 & 1.000 & 80.4 & 0.577 & 0.304 & 0.317 & 1.71 & 76 & 207 & 0.526 & & 0.969 & $1154 \pm 93$ & $1.327 \pm 0.187$ & 0.73 & 0.840 & 10.92 \\
\hline \multicolumn{18}{|c|}{ (b) Best-fit model with bloated secondary star } \\
\hline 4 & 139 & 1.120 & 80.5 & 0.568 & 0.235 & 0.290 & 1.70 & 72 & 192 & 0.413 & 1.217 & & $1054 \pm 85$ & $1.217 \pm 0.172$ & 1.00 & 1.150 & 9.20 \\
\hline \multicolumn{18}{|c|}{ (c) Models with bloated secondaries, delineating the $90 \%$ confidence limits } \\
\hline 5 & 139 & 1.035 & 80.0 & 0.600 & 0.281 & 0.309 & 1.74 & 68 & 195 & 0.469 & 1.117 & & $1127 \pm 91$ & $1.296 \pm 0.183$ & 0.86 & 0.991 & 10.00 \\
\hline 6 & 149 & 1.135 & 81.4 & 0.524 & 0.224 & 0.285 & 1.64 & 83 & 202 & 0.427 & 1.190 & & $1037 \pm 84$ & $1.192 \pm 0.168$ & 1.00 & 1.148 & 9.20 \\
\hline 7 & 129 & 1.095 & 79.7 & 0.617 & 0.247 & 0.297 & 1.77 & 62 & 183 & 0.401 & 1.244 & & $1081 \pm 88$ & $1.244 \pm 0.175$ & 1.00 & 1.150 & 9.20 \\
\hline 8 & 139 & 1.190 & 81.1 & 0.541 & 0.201 & 0.275 & 1.66 & 75 & 189 & 0.371 & 1.313 & & $1002 \pm 81$ & $1.152 \pm 0.162$ & 1.14 & 1.310 & 8.60 \\
\hline
\end{tabular}

Notes. The columns contain $K_{2}^{\prime}(2)$, the bloating factor $f_{3}$ (3), the derived model parameters (3-11), the model radius of the He-core of CO-core WD of the mass of Col. (11) cooled to $10 \mathrm{kK}(12,13)$, the distance (14), the spectroscopically derived WD radius (15), the ratio of the radii (16), the angular radius of the WD (17), and its implied photospheric temperature (18).

errors refer to the $90 \%$ confidence level and include besides the statistical also the systematic error caused by the remaining uncertainty in the level of Stark broadening (Sect. 6.3). Quadratically adding the error from an estimated $10 \%$ uncertainty in the flux calibration of the spectropolarimetry gives $R_{\mathrm{wd}} / d=$ $(1.15 \pm 0.14) \times 10^{9} \mathrm{~cm} \mathrm{kpc}^{-1}$. The error budget of $R_{\mathrm{wd}}$ includes in addition the $8.1 \%$ uncertainty in the mean dM5-dM6 distance from Sect. 5.2, raising the error of $R_{\mathrm{wd}}$ to $14.1 \%$. Despite its large error, the measured radius proves helpful in determining the system parameters in Sect. 7.5.

The photospheric temperature of the WD in HY Eri is lower than in practically all other well-studied polars (Townsley \& Gänsicke 2009). The low temperature is directly related to the low observed equivalent width $W_{\text {obs }}$ of the Zeeman lines in HY Eri. The equivalent width in the theoretical Zeeman spectra has its peak at $T_{\text {eff }}=12 \mathrm{kK}$ and drops rapidly toward lower and higher temperatures. $W_{\text {obs }}$ falls by a factor of 3.2 below the peak value for nominal Stark broadening. Fitting $W_{\text {obs }}$ and the spectral slope simultaneously, requires $T_{\mathrm{ph}}$ significantly below $12 \mathrm{kK}$ and $T_{\mathrm{sp}} \gg 12 \mathrm{kK}$. The fit deteriorates with rising $T_{\mathrm{ph}}$ and becomes unacceptably bad at $11 \mathrm{kK}$, even allowing for a variation in the level of Stark broadening.

\section{System parameters}

\subsection{Narrow emission lines as tracers of the motion of the secondary star}

Polars feature narrow emission lines of hydrogen, helium and metals that are thought to originate on the irradiated face of secondary star. In some polars, however, the radial velocity amplitudes of individual lines differ. Helium lines with their high ionization potential show lower amplitudes than hydrogen, while the low-ionization near infrared Ca II lines have the greatest and rather stable amplitudes (Schwope et al. 2000, 2011; Schwope \& Christensen 2010). Obviously, the distribution of the emission differs between individual lines, with the helium lines probably originating, in part, from structures outside the chromosphere of the star, such as coronal prominences. Modeling is straightforward as long as the emission originates from locations geometrically close to the surface of the star, which appears to be the case for the low-ionization metal lines and, in some polars, for hydrogen lines. Describing the line emission over the secondary star requires either a dedicated theoretical model or an empirical ansatz that derives the distribution from unfolding highly resolved observed line profiles.

\subsection{Models of the irradiated secondary star}

We calculated the radial velocity amplitude $K_{2}^{\prime}$ of the narrow emission line, considering a Roche-lobe filling star that is irradiated by a source at the position of the WD. Each surface element receives an incident flux $f_{\text {in }} \propto \cos \vartheta / \delta^{2}$, with $\vartheta$ the angle of incidence and $\delta$ the distance from the source. In response, it emits a line flux $f_{\text {line }}$ that varies with $f_{\text {in }}$, but may depend on additional parameters. For a given model, we calculated synthetic emission line spectra and determined the lever arm of the line emission region $a_{\mathrm{irr}}(q)=a_{2} K_{2}^{\prime} / K_{2}$ as a function of the mass ratio $q=M_{2} / M_{1}$, with $a_{2}=1 /(1+q)$. Below, we quote polynomial approximations for $a_{\mathrm{irr}}(q)^{10}$.

The irradiation model of Beuermann \& Thomas (1990; henceforth BT90), equates the line intensity emitted from a surface element to a fraction of the total incident flux, $f_{\text {line }} \propto$ $\cos \vartheta / \delta^{2}$. The emitted intensity drops from a maximum at $L_{1}$, where $\vartheta \simeq 40^{\circ}$, down to zero at the terminator of the irradiated region, where $\vartheta=90^{\circ}$. Hence, BT90 favors emission from regions near $L_{1}$. The modified version BT90m uses $f_{\text {line }} \propto$ $(\cos \vartheta)^{m} / \delta^{2}$, with $m$ a heuristic free parameter. This modification was motivated by the study of irradiated WD atmospheres by König et al. (2006, their Sect. 4.2), who found that the narrow emission cores of Ly $\alpha$ increased drastically when $\vartheta$ approached $90^{\circ}$ and the incident energy was deposited increasingly higher up in the atmosphere. There is no simple way to relate $m$ to physics, however. An entirely different approach was taken in Beuermann $\&$ Reinsch (2008, their Sects. 5.4, 6.1, and Fig. 10), where we determined the distribution of the Ca II $\lambda 8498$ emission as a function of $\vartheta$ empirically by unfolding the high-resolution line profiles of the intermediate polar EX Hya. A parameterized form of the Ca II emission model was implemented in our model BR08. It provides an internally consistent description of the emission of Ca II $\lambda 8498$ and numerous other metal lines that share the motion of CaII. The model gives larger weight to surface

10 Model BR08: $a_{\mathrm{irr}}=0.806155-0.959167 q+0.356060 q^{2}$; Model BT90: $a_{\text {irr }}=0.786470-0.978076 q+0.368182 q^{2}$; both for $q=$ $0.35-0.80$. 

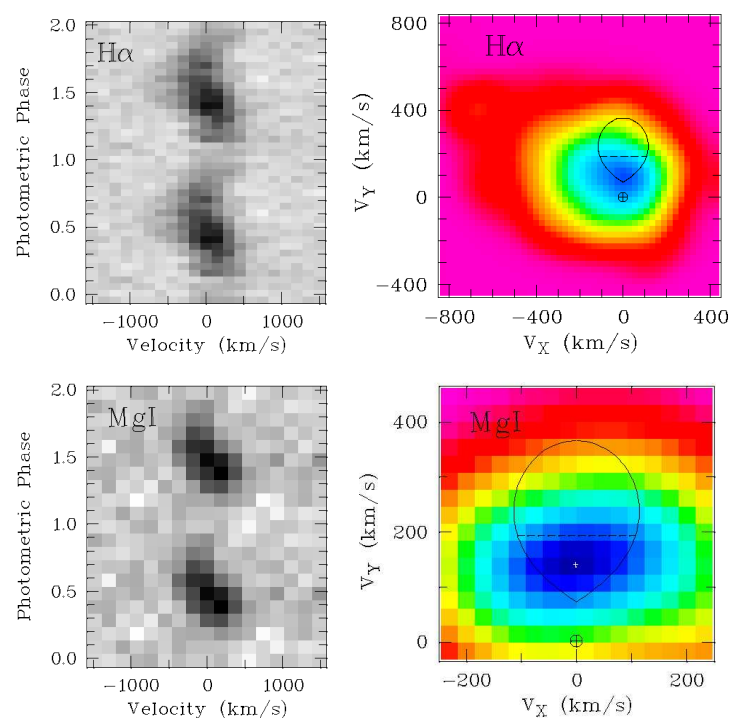

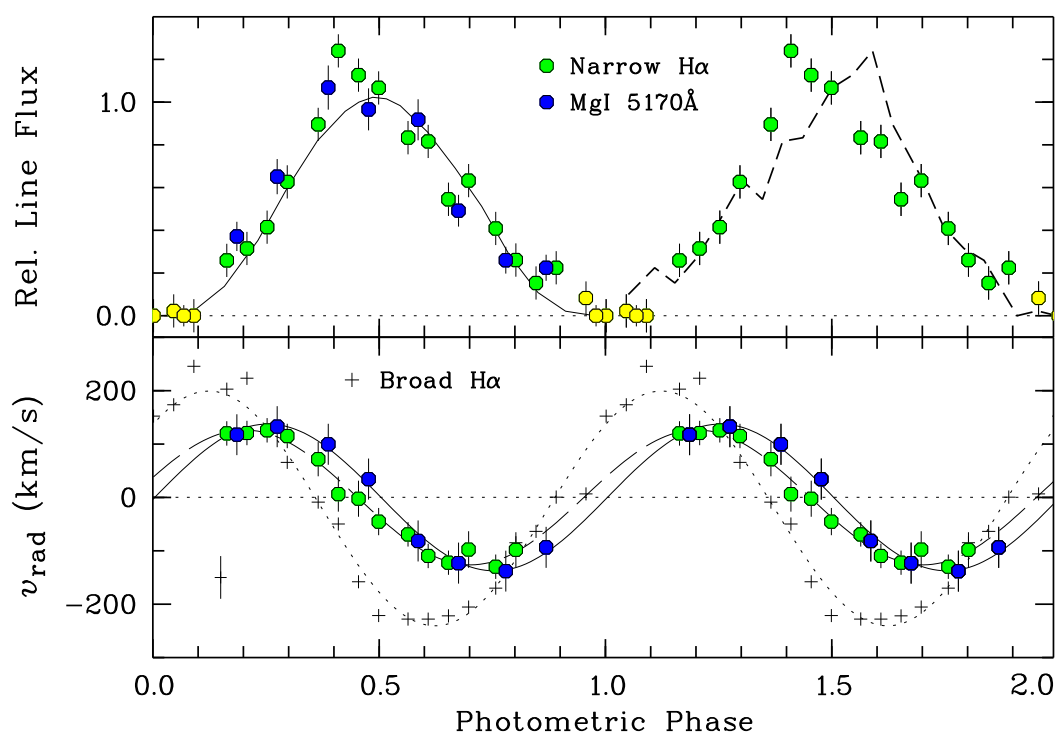

Fig. 9. Left: $\mathrm{H} \alpha$ and $\mathrm{Mg} \mathrm{I} \lambda 5170$ emission lines derived from the spectropolarimetry of $31 \mathrm{Dec} 2008$, with the neighboring continuum subtracted and shown twice for better visibility. Center: corresponding Doppler maps computed by the maximum entropy method MEM. The overlays show the Roche lobes of the secondary for the best bloated model of Sect. 7.5. Top right: the first orbit shows the orbital flux variations of the Mg I line (blue) and the narrow component of $\mathrm{H} \alpha$ (green). The second orbit shows the $\mathrm{H} \alpha$ flux and its mirror image around $\phi=0.5$ (see text). Bottom right: radial velocity curves of the $\mathrm{MgI}$ line (blue), the narrow $\mathrm{H} \alpha$ component (green), and the broad $\mathrm{H} \alpha$ component (crosses). A typical error bar for the broad line is shown in the lower left.

elements near the terminator, equivalent to moderate limb brightening. To put the models into perspective, we note that BR08 corresponds approximately to BT90m with $m \simeq 0.3$. BT90 and BR08 bracket about the full range of possible irradiation scenarios of the atmosphere of the secondary. Only models with still more pronounced limb brightening, equivalent to BT90m with $m<0.3$, would yield a still larger value of $a_{\text {irr }}$ (and a still smaller primary mass). An estimate of the remaining systematic errors is given in Sect. 7.5.

\subsection{Narrow emission lines in HY Eri}

In its 2008 low state, HY Eri displayed emission lines of hydrogen, HeI, and $\mathrm{MgI}$ I5170. The near infrared CaII triplet was not detectable against the cyclotron background. In $\mathrm{H} \alpha$, the broad component with a FWHM of $23 \AA$ and the unresolved narrow component can be separated at our $10 \AA$ spectral resolution, while this becomes infeasible for the higher Balmer lines, which are embedded in complex Zeeman absorption troughs. $\mathrm{Mg} \mathrm{I} \lambda 5170$ is not disturbed and is the only metal line that is sufficiently strong for a radial velocity study. The left panels in Fig. 9 show pseudo-trailed spectra of $\mathrm{H} \alpha$ and $\mathrm{Mg} \mathrm{I} \lambda 5170$ with the phase-dependent continuum subtracted. The gray scale is inverted compared with Fig. 5. We used spectral set 2 with 20 spectra per orbit for $\mathrm{H} \alpha$ and set 1 with 10 spectra per orbit for the weaker $\mathrm{Mg}$ I line. The two orbits were folded and the data shown twice for better visibility.

The upper right panel in Fig. 9 shows the orbital flux variations of $\mathrm{MgI} \lambda 5170$ (blue) and of the narrow $\mathrm{H} \alpha$ component (green). Maximum flux occurs near $\phi=0.5$, when the illuminated hemisphere is in view. Half an orbit later, the $\mathrm{Mg}$ line disappears and the narrow $\mathrm{H} \alpha$ component can no longer be discriminated against the underlying broad component (yellow). The upper half of the light curve is skewed, as is illustrated in the second orbit, where the $\mathrm{H} \alpha$ light curve is compared with its own mirror image relative to $\phi=0.5$ (dashed curve). The skew is, at least in part, due to statistical fluctuations between the two orbits. Apart from this, the light curve is well described by the irradiation model BR08 (solid curve). The lower right panel of Fig. 9 shows the radial velocity curves of $\mathrm{MgI} \lambda 5170$ (blue), of the narrow component of $\mathrm{H} \alpha$ (green), and of the broad $\mathrm{H} \alpha$ emission (crosses). Although $\mathrm{MgI} \lambda 5170$ is weaker than $\mathrm{H} \alpha$, the radial velocities have similar errors because the former were derived from single-Gaussian and the latter from the more uncertain double-Gaussian fits. $\mathrm{MgI} \lambda 5170$ has a radial velocity amplitude of $K_{2}^{\prime}=139 \pm 10 \mathrm{~km} \mathrm{~s}^{-1}$ with a blue-to-red zero-crossing phase of $\phi_{0}=0.02 \pm 0.02$, the narrow $\mathrm{H} \alpha$ line has $K_{2}^{\prime}=125 \pm 9 \mathrm{~km} \mathrm{~s}^{-1}$ with $\phi_{0}=-0.06 \pm 0.02$. The broad component has a velocity amplitude $K_{\text {broad }}=220 \pm 14 \mathrm{~km} \mathrm{~s}^{-1}$ with $\phi_{0}=-0.13 \pm 0.02$ and $\gamma=-21 \pm 9 \mathrm{~km} \mathrm{~s}^{-1}$ relative to the narrow component. All errors refer to the $90 \%$ confidence level.

We investigated the origin of the lines by calculating Doppler tomograms, using the maximum entropy method MEM (Spruit 1998; Marsh \& Schwope 2016). Given the small number of phase intervals, the tomograms are sensitive to noise and have been slightly smoothed with a velocity filter corresponding to 0.3 spectral resolution elements. The resulting tomograms are shown in the center panels of Fig. 9, with the outline of the Roche lobe of the secondary for our best-fitting dynamical model overplotted (Table 9, line 4). The bulk of the emission can be uniquely allocated to the illuminated face of the secondary star and the vicinity of the inner Lagrangian point $L_{1}$. The rainbow color scale ranges from blue for the highest intensity down to red. Overall, the $\mathrm{H} \alpha$ tomogram is tilted toward the leading hemisphere of the secondary, with the asymmetry related to the finite negative $\phi_{0}$ and the existence of the underlying broad component. The latter with its best visibility at $\phi=0.60$ is represented by the tail that extends to $V_{X}=-700 \mathrm{~km} \mathrm{~s}^{-1}$ and $V_{Y}=+450 \mathrm{~km} \mathrm{~s}^{-1}$. This direction differs significantly from that of the standard ballistic stream seen in many polars in their high states, which moves in velocity space from $L_{1}$ to large negative $V_{X}$ at nearly constant $V_{Y}$. A tail at similarly odd velocities was seen in the He II $\lambda 4686$ tomogram of AM Her (Staude et al. 2004) and tentatively interpreted in terms of a non-standard accretion 
stream that couples from the secondary immediately to a polar field line of the WD. This is an intriguing proposition in view of our suggestion in Sect. 6.4 that pole 2 of HY Eri is fed by such a scenario. In view of these idiosyncrasies, we should be wary of interpreting the narrow $\mathrm{H} \alpha$ line in HY Eri as of purely chromospheric origin.

The Mg I $\lambda 5170$ line is free of the complications by a broad component, the tomogram looks more regular, and the phase of zero radial velocity is consistent with inferior or superior conjunction of the secondary star. The bottom center panel of Fig. 9 shows the enlarged central portion of the tomogram. The emission is centered on the illuminated part of the star, with the peak intensity occurring at a $Y$-velocity that agrees with $K_{2}^{\prime}=139 \pm 10 \mathrm{~km} \mathrm{~s}^{-1}$ obtained from the radial-velocity analysis (small white cross).

\subsection{Mass-radius relation of the secondary star}

Deriving stellar masses requires that we adopt a mass-radius relation $R_{2}\left(M_{2}\right)$ for the Roche-lobe filling secondary star. We used theoretical models by Baraffe et al. (1998, 2015, henceforth $\mathrm{BCAH}$ and BHAC) for main sequence stars of solar composition evolved to $1 \mathrm{Gyr}$. This is the approximate cooling age of the WD in HY Eri, discounting compressional heating by accretion, and the minimal age of the secondary. For ease of application, we represented the radii by power laws $R_{2}\left(M_{2}\right)^{11}$ Since secondary stars in CVs are known to be more or less bloated compared with field stars, we considered radii expanded over those of the BHAC models by the following processes: (i) magnetic activity and spot coverage (Chabrier et al. 2007; Morales et al. 2010; Knigge et al. 2011; Parsons et al. 2018); (ii) tidal and rotational deformation of Roche-lobe filling stars (Renvoizé et al. 2002); and (iii) inflation by magnetic braking that drives the star out of thermal equilibrium (Knigge et al. 2011). Effect (i) describes the radius excess that compensates for the reduced radiative efficiency caused by starspots. Knigge et al. (2011) and Parsons et al. (2018) found a mean excess of $5 \%$ for stars with mass below $0.35 M_{\odot}$. Morales et al. (2010) and Knigge et al. (2011) argued that high-latitude spots may mimic a larger radius in certain eclipsing binaries, accounting for $3 \%$ of the excess. Proceeding conservatively, we accept this argument and adopted a bloating factor $f_{1}=1.020$. A Roche-lobe filling star in a short period binary can not escape effect (ii), which increases the radius by a factor $f_{2}=1.045$ independent of $q$ (Renvoizé et al. 2002; Knigge et al. 2011). Effect (iii) is described by a free factor $f_{3}$ that may range from unity up to about 1.30. The adopted stellar radii $R_{2}=f_{1} f_{2} f_{3} R_{\mathrm{BHAC}}$ are fully consistent with those employed by Knigge et al. (2011) in their evolutionary sequences. Using the models of stars with solar composition evolved to ages of 5 or $10 \mathrm{Gyr}$ instead of $1 \mathrm{Gyr}$, the dynamical solution yields WD masses lower by $3 \%$ or $5 \%$, respectively. For a metal-poor secondary with $[\mathrm{M} / \mathrm{H}]=-1$, the masses would be higher by $8 \%$ at $1 \mathrm{Gyr}$, but correspondingly lower again for larger ages.

\subsection{Component masses and distance}

For a given irradiation model and a mass-radius relation of the secondary star, we obtained the system parameters that match the radial-velocity amplitude $K_{2}^{\prime}$ and the eclipse duration $\Delta t_{\mathrm{ecl}}$.

${ }^{11}$ Baraffe et al. (2015) main sequence mass-radius relations, $M_{2} / M_{\odot}=$ $0.15-0.40 M_{\odot}, M H=0,1 \mathrm{Gyr}: R_{2} / R_{\odot}=0.736\left(M_{2} / M_{\odot}\right)^{0.761} ; 5 \mathrm{Gyr}$ : $R_{2} / R_{\odot}=0.766\left(M_{2} / M_{\odot}\right)^{0.780} ; 10 \mathrm{Gyr}: R_{2} / R_{\odot}=0.787\left(M_{2} / M_{\odot}\right)^{0.792}$; $M H=-1,1$ Gyr: $R_{2} / R_{\odot}=0.735\left(M_{2} / M_{\odot}\right)^{0.793}$.

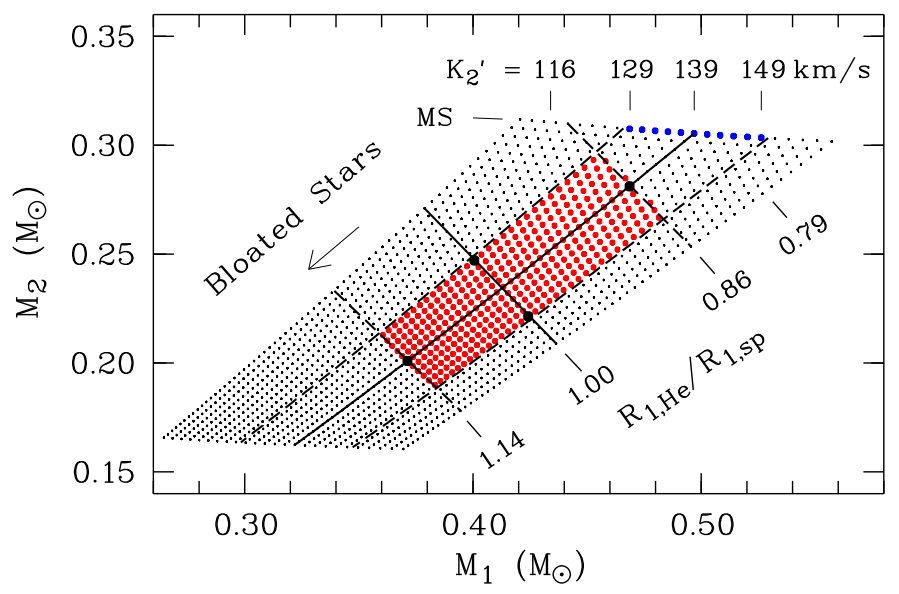

Fig. 10. Dynamic models of HY Eri in the $M_{1}-M_{2}$ plane. The irradiation model is BR08, $K_{2}^{\prime}$ ranges from 111 to $159 \mathrm{~km} \mathrm{~s}^{-1}$ in steps of $2 \mathrm{~km} \mathrm{~s}^{-1}$, and the bloating factor $f_{3}$ ranges from 1.000 to 1.300 in steps of 0.005 . The models with main sequence (MS) secondaries are marked by blue dots. Red dots denote the models that match both, the measured radial-velocity amplitude of $\mathrm{Mg} \mathrm{L} \lambda 5170$ and the spectroscopically measured WD radius within their $90 \%$ confidence errors.

We adopted $K_{2}^{\prime}=139 \pm 10 \mathrm{~km} \mathrm{~s}^{-1}$ of the MgI $\lambda 5170$ line and BR08 as the standard. Results are presented in Table 9 and Fig. 10. The derived parameters include the masses and radii of the components, the distance $d$ obtained from the angular radius of the secondary star (Sect. 5.2) and the spectroscopic radius $R_{1, \text { sp }}$ of the WD obtained from $d$ and the angular radius of the WD (Sect. 6.5). The listed model radii in Cols. (12) and (13) of Table 9 refer to He-core and CO-core WDs with thick hydrogen envelopes ${ }^{12}$ evolved to $T_{\text {eff }}=10 \mathrm{kK}$ (Panei et al. 2007; Renedo et al. 2010; Althaus et al. 2013) ${ }^{13}$. The radii for an effective temperature of $9 \mathrm{kK}$ are only minimally smaller.

To start with, we take the secondary to be an unbloated main sequence star with $f_{3}=1.0$, an assumption that yields the maximum primary mass, but disregards the spectroscopic information from Sect. 6.5. For the $\mathrm{Mg}$ line with $K_{2}^{\prime}=139 \pm 10 \mathrm{~km} \mathrm{~s}^{-1}$ and BR08, the component masses are $M_{1}=0.497 \pm 0.029 M_{\odot}$ and $M_{2}=0.305 \pm 0.002 M_{\odot}$ (Table 9 , lines 1 to $3,90 \%$ confidence errors). Interestingly, the $\mathrm{H} \alpha$ value $K_{2}^{\prime}=125 \pm 9 \mathrm{~km} \mathrm{~s}^{-1}$ and BT90, yield practically the same primary mass, $M_{1}=$ $0.487 \pm 0.026 M_{\odot}$, but this combination lacks the internal consistency that exists between the Mg I line and the metal-line calibrated model BR08. Cross-combining velocity amplitude and irradiation model gives an indication of the remaining systematic error: $\mathrm{H} \alpha$ and BR08 give $M_{1}=0.458 M_{\odot}, \mathrm{Mg} \mathrm{I}$ and BT90 give $M_{1}=0.528 M_{\odot}$, or combined $M_{1}=0.493 \pm 0.035 M_{\odot}$. Hence the so-defined systematic error is of the same size as the statistical error of $0.029 M_{\odot}$. In summary, the assumption of a main sequence secondary identifies the primary either as a He-core WD or a CO-core WD very close to its minimum mass of $0.53 \pm 0.02 M_{\odot}$ (Moehler et al. 2004; Kalirai et al. 2008). The fault with the main sequence assumption is the neglect of the spectroscopic evidence of Sect. 6.5 on the angular radius and the effective temperature of the WD. The implied model radius of the primary in either Cols. (12) or (13) of Table 9, lines 1-3, falls far short of the spectroscopically determined radius $R_{1, \mathrm{sp}}$ in Col. (15), calculated from $R_{\mathrm{wd}} / d=(1.15 \pm 0.14) \times 10^{9} \mathrm{~cm} \mathrm{kpc}^{-1}$

\footnotetext{
12 The envelope mass varies between $M_{\mathrm{H}}=8 \times 10^{-5}$ at $M_{1}=0.52 M_{\odot}$ and $3 \times 10^{-4} M_{\odot}$ at $0.3 M_{\odot}$, staying below the respective ignition masses. 13 http : //evolgroup. fcaglp. unlp . edu . ar/TRACKS/DA . html
} 
(Sect. 6.5) and the $R_{2}$-dependent distance $d$ in Col. (14), where we have added the errors quadratically. The employed angular radius of the WD belongs to the best-fit photospheric temperature $T_{\mathrm{ph}}=9.2 \pm 0.7 \mathrm{kK}$. Had the WD the radius of Cols. (12) or (13), the observed spectral flux would demand that its temperature would be that in Col. (18). As noted in Sect. 6.5, a decent Zeeman spectral fit can not be achieved for $T_{\mathrm{ph}}>10 \mathrm{kK}$, further reducing the probability that the primary in HY Eri is a low-mass CO WD.

In a second step, we considered models with bloated secondary stars. We calculated a grid of models with radial velocity amplitudes $K_{2}^{\prime}=110$ to $160 \mathrm{~km} \mathrm{~s}^{-1}$ and expansion factors $f_{3}=$ 1.0 to 1.3 in steps of $2 \mathrm{~km} \mathrm{~s}^{-1}$ and 0.005 , respectively. We identified $K_{2}^{\prime}$ with the radial velocity amplitude of the $\mathrm{Mg} \mathrm{I} \lambda 5170$ line and converted it to $K_{2}$, using the BR08 model. The resulting component masses are depicted in Fig. 10, where each model is represented by a dot. The main sequence models considered above are marked in blue. Models that comply with the MgI amplitude $K_{2}^{\prime}=139 \pm 10 \mathrm{~km} \mathrm{~s}^{-1}$, are located between the two dashed lines, extending from the upper right to the lower left. Along this path, the bloating factor $f_{3}$ of the secondary star increases, the mass of the WD decreases, its radius increases, and its temperature decreases. Models with He-core WDs, whose radii agree within the uncertainties with the spectroscopically determined WD radius, $R_{1, \mathrm{He}} / R_{1, \mathrm{sp}}=1.00 \pm 0.14(90 \%$ confidence error, Sect. 6.5) are located between the two dashed lines that run from the upper left to the lower right, and models that match both conditions are marked by red dots. The optimal dynamical model in line 4 of Table 9 corresponds to the intersection of the two solid lines and the WD parameters at this point correspond to those of the optimal multipole Zeeman fit in Table 8. Lines 5-8 of Table 9 contain the model parameters for the four cardinal points of the red-dotted region, marked by the four black dots. Column (3) of the table lists the bloating factor $f_{3}$. Bloating ranges from a minimal $3.5 \%$ to $19 \%$, indicating that the secondary is only moderately expanded as may be expected for a polar that experiences reduced magnetic braking (Wickramasinghe \& Wu 1994; Webbink \& Wickramasinghe 2002). As discussed in Sect. 8, minimal bloating that goes along with a low accretion rate is also required to explain the low photospheric temperature of the WD in terms of compressional heating (Townsley \& Gänsicke 2009). At the optimal position and at two of the cardinal points, the model and observed WD radii in Cols. (12) and (15) agree. At the two other cardinal points, they disagree by the permitted $\pm 14 \%$ (Col. 16). The $90 \%$ confidence region for the combined dynamical and spectroscopic fit is defined by a quasi-ellipse that is inscribed to the red-dotted quadrilateral and passes through the cardinal points (not shown). It limits the component masses to $M_{1}=0.413+0.058,-0.044 M_{\odot}$ and $M_{2}=0.235+0.048,-0.038 M_{\odot}(90 \%$ confidence errors $)$, or $M_{1}=0.42 \pm 0.05 M_{\odot}$ and $M_{2}=0.24 \pm 0.04 M_{\odot}$, with $q$ between 0.51 and 0.63 . The combined dynamical and spectroscopic fit identifies the primary in HY Eri as a low-mass WD, consistent with having a helium core. The mass of the secondary is normal for a CV with an orbital period of $2.855 \mathrm{~h}$. It may be fully convective or retain a radiative core, depending on its prehistory (Knigge et al. 2011).

The observed parameters of HY Eri are summarized in Table 10. At a distance $d=1050 \pm 110 \mathrm{pc}$ and a galactic latitude $b=-26.1^{\circ}$, it is located close to $500 \mathrm{pc}$ below the galactic plane. The second Gaia data release (Gaia Collaboration 2018) yielded a parallax $\pi=-0.1 \pm 0.8$ mas at a mean $g=20.3$, or a distance of $d>830$ pc (Bailer-Jones et al. 2018), consistent with all entries in Table 9.
Table 10. System parameters of HY Eri with $90 \%$ confidence errors.

\begin{tabular}{|c|c|c|}
\hline Parameter & Value & Error \\
\hline Orbital period $P_{\text {orb }}(\mathrm{s})$ & 10278.9 & \\
\hline Eclipse duration $F W H M \Delta t_{\mathrm{ecl}}(\mathrm{s})$ & 910.6 & 1.5 \\
\hline Primary mass $M_{1}\left(M_{\odot}\right)$ & 0.42 & 0.05 \\
\hline Primary radius $R_{1}$ at $T_{\mathrm{ph}}\left(10^{9} \mathrm{~cm}\right)$ & 1.22 & 0.10 \\
\hline Secondary mass $M_{2}\left(M_{\odot}\right)$ & 0.24 & 0.04 \\
\hline Secondary radius $R_{2}\left(R_{\odot}\right)$ & 0.29 & 0.02 \\
\hline Mass ratio $q$ & 0.57 & 0.06 \\
\hline Binary separation $a\left(10^{10} \mathrm{~cm}\right)$ & 6.16 & 0.28 \\
\hline Inclination $i\left(^{\circ}\right)$ & 80.6 & 0.9 \\
\hline Distance $d(\mathrm{pc})$ & 1050 & 110 \\
\hline$T_{\text {eff }}$ of secondary $(\mathrm{K})$ & 3000 & 100 \\
\hline Luminosity of secondary $L_{\mathrm{bol}}\left(10^{31} \mathrm{erg} \mathrm{s}^{-1}\right)$ & 2.3 & 0.6 \\
\hline$T_{\mathrm{eff}}$ of photosphere of WD $T_{\mathrm{ph}}(\mathrm{kK})$ & 9.2 & 0.7 \\
\hline$T_{\text {eff }}$ of pole cap of WD $T_{\text {cap }}(\mathrm{kK})$ & 14.2 & 1.0 \\
\hline Weighted mean of $T_{\mathrm{ph}}$ and $T_{\mathrm{cap}}(\mathrm{kK})$ & 10.1 & 0.9 \\
\hline$T_{\text {eff }}$ of spot 1 of WD $T_{\mathrm{sp}}(\mathrm{kK})$ & $78.0^{(1)}$ & 8.0 \\
\hline Magnetic field strength in spot $1 B_{\mathrm{sp}, 1}(\mathrm{MG})$ & 27.4 & 0.2 \\
\hline Magnetic field strength in spot $2 B_{\mathrm{sp}, 2}(\mathrm{MG})$ & 28.7 & 0.3 \\
\hline
\end{tabular}

Notes. (1) Subject to the spot size.

\subsection{Luminosity and accretion rate}

The spectral energy distribution (SED) in Fig. 11 provides an overview of the long-term variability of HY Eri. It shows the spectrum of 1993 from Fig. 3 (black curve), that of 2008 from Fig. 7 (red), and the eclipse spectrum from the center panel of Fig. 2 (blue). The photometric data were accessed via the Vizier SED tool provided by the $\mathrm{CDS}^{14}$. They include the $\mathrm{SDSS}^{15}$, the UKIDSS, and the Wide-field Infrared Survey (WISE; Cutri et al. 2013) as cyan blue dots, the Two Micron All Sky Survey (2MASS; Skrutskie et al. 2006) as green dots, the Galaxy Evolution Explorer (GALEX; Martin et al. 2005; Bianchi et al. 2011) as blue dots, and the catalogs PPMXL (Roeser et al. 2010) and NOMAD (Zacharias et al. 2005) as red dots. The range of the MONET WL measurements of Fig. 1 is indicated by the four open triangles that refer to the observations of January 2010, November 2010, 2017/18, and the remnant flux inside the eclipse. They delineate the same range of flux levels as the independent photometric and spectrophotometric observations. The secondary star $(+)$ represents a lower flux limit in the red part of the SED.

To a first approximation, the high state is characterized by a roughly flat SED from the infrared to the FUV, with $F_{v} \simeq 0.5 \mathrm{mJy}$ and an integrated energy flux of $F_{\mathrm{UV} \text {,opt }} \simeq 1.5 \times$ $10^{-11} \mathrm{erg} \mathrm{cm}^{-2} \mathrm{~s}^{-1}$. At a distance of $1 \mathrm{kpc}$, the high-state UVoptical luminosity amounts to $L_{\mathrm{UV}, \mathrm{opt}} \simeq 2.0 \times 10^{33} \mathrm{erg} \mathrm{s}^{-1}$ for emission into $4 \pi$. The $2002 \mathrm{UV}$ and visual photometry with the XMM-Newton optical monitor demonstrates that the simultaneous X-ray observation was also taken in a near-high state. The bolometric X-ray flux measured in the ROSAT and XMMNewton observations is not well established. In Sect. 4 we quoted a conservative high-state level of the bolometric soft X-ray flux of $F_{\mathrm{X}} \simeq 1.5 \times 10^{-11} \mathrm{erg} \mathrm{cm}^{-2} \mathrm{~s}^{-1}$, although the X-ray flux may have been significantly higher at times. Hence, as a conservative

\footnotetext{
${ }^{14}$ Centre de Données astronomiques de Strasbourg, http://vizier. u-strasbg.fr/vizier/sed/

15 Data Release 15, http://www . sdss.org/dr15
} 
K. Beuermann et al.: Neglected X-ray discovered polars. II.

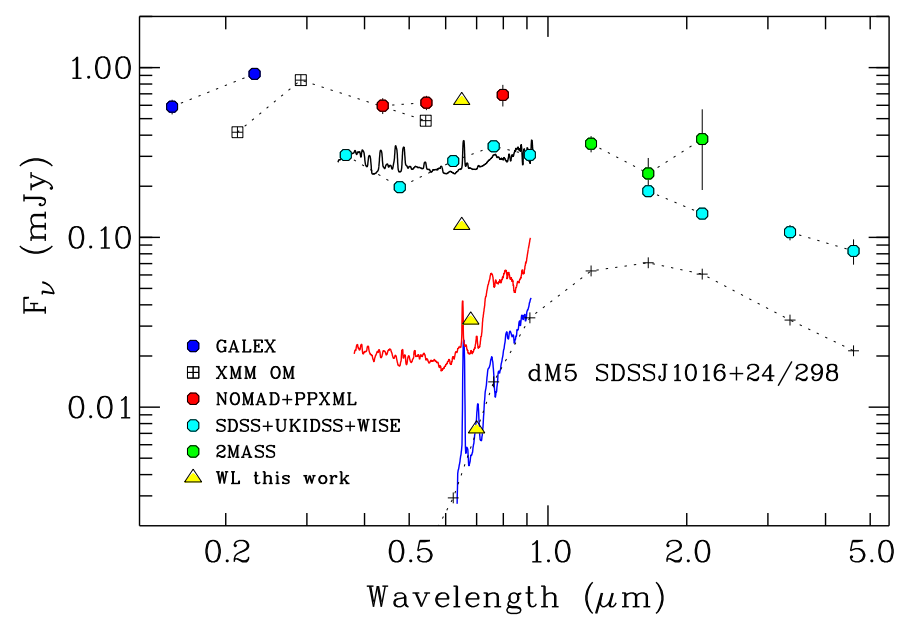

Fig. 11. Spectral energy distribution of HY Eri from the far ultraviolet to the near infrared based in part on publicly available photometric data accessed via the Vizier SED tool available at the CDS in Strasbourg.

estimate the total high-state luminosity, not accounting for the hard X-ray region, was $L_{\mathrm{X}, \mathrm{UV} \text {,opt }} \simeq 4 \times 10^{33} \mathrm{erg} \mathrm{s}^{-1}$. For the bestfit model in Table 9, line 4 and Table 10 with a WD of $0.42 M_{\odot}$, this luminosity requires an accretion rate of $\dot{M} \simeq 9 \times 10^{16} \mathrm{~g} \mathrm{~s}^{-1}=$ $1.4 \times 10^{-9} M_{\odot} \mathrm{yr}^{-1}$, On the other hand, the integrated orbital mean cyclotron flux in the 2008 low state, including the extrapolation into the unobserved infrared regime, amounted to $F_{\text {opt,IR }} \simeq$ $3 \times 10^{-14} \mathrm{erg} \mathrm{cm}^{-2} \mathrm{~s}^{-1}$. The implied remnant accretion rate was a mere $\dot{M} \simeq 1.4 \times 10^{-12} M_{\odot} \mathrm{yr}^{-1}$, three orders of magnitude lower than in the high state.

\section{Discussion}

HY Eri belongs to the rather small group of about a dozen eclipsing polars that may be considered well studied (Ritter \& Kolb 2003, final edition 7.24, 2016). When looked at superficially, it is a rather uninspiring member of the class, being distant and faint, and lapsing into prolonged states of low accretion ${ }^{16}$. The present study, however, reveals HY Eri as a system that is peculiar in several respects and in part unique among polars.

We identified it as a permanent two-pole accretor with both poles being active for accretion rates differing by three orders of magnitude. Two nearly opposite accretion spots with similar field strengths seem to suggest a dipolar field, but that is probably an illusion. Our combined quasi-dipole fit to the spectra of both poles is formally good and yields field directions in both accretion spots that are consistent with the results of our cyclotron line fits, but nevertheless the magnetic geometry is characterized by tightly closed field lines in both spots that seem to preclude accretion. In an extensive grid search, we discovered an alternative close-to-perfect fit for a field structure with a polar field strength of the dipole $B_{\text {dip }}=40.4 \mathrm{MG}$ and relative field strengths of quadrupole and octupole $B_{\text {qua }} / B_{\text {dip }}=\mp 0.14$ and $B_{\text {oct }} / B_{\text {dip }}=-0.44$. It possesses open field lines in both spots, allowing the southern spot 1 to accrete from the nearby orbital plane, while the field in the northern spot 2 leads over the rotational pole to a place somewhere near the secondary star. If that path is active, it obviates the need for the plasma accreted at spot 2 to travel halfway around the WD until it attaches to a

\footnotetext{
16 A long-term light curve is available from the Catalina Sky Survey at http://nesssi.cacr.caltech.edu/catalina/CVservice/ cVtable.html
}

near-polar field line. We caution, however, that models that include the full zoo of tesseral harmonics may favor still different magnetic geometries. Furthermore, Zeeman fits based on intensity spectra alone may not yield a unique result. The simultaneous fit to intensity and circular polarization spectra offers better perspectives as demonstrated by Euchner et al. (2002, 2005, 2006) and Beuermann et al. (2007).

The Zeeman spectra of 2008 were obtained in a state of low accretion and yielded a mean mass-flow rate $\dot{m}$ close to that of the bombardment solution of Woelk \& Beuermann (1992). The spot fields obtained from the Zeeman and cyclotron fits differ by about $3 \%$, suggesting that the cyclotron emission originates at a height above the photosphere of roughly $0.01 R_{\mathrm{wd}}$, provided both originate at the same position on the WD. This altitude is in the same ballpark as the shock height of $\sim 0.006 R_{\mathrm{wd}}$ predicted by two fluid cooling theory (Fischer $\&$ Beuermann 2001) for the mass flow rate listed in Table 6.

The property by which HY Eri deviates most drastically from other polars, is the low primary mass of $M_{\mathrm{wd}}=0.42 \pm 0.05 M_{\odot}$, based on the simultaneous dynamical and Zeeman spectral analysis. The dynamical analysis alone limits the primary mass to $M_{1}<0.53 M_{\odot}$. The secondary mass of $M_{2}=0.24 \pm$ $0.04 M_{\odot}$ appears normal for a CV of $2.855 \mathrm{~h}$ orbital period. Only two other polars in the Ritter \& Kolb catalog (Ritter \& Kolb 2003, final edition 7.24, 2016) have reported WD masses below $0.50 M_{\odot}$. Gänsicke et al. (2000) derived the angular radius of the WD in V1043 Cen from far-ultraviolet spectroscopy and the radius and mass from a distance estimate of $200 \mathrm{pc}$. At the Gaia distance of 172 pc (Gaia Collaboration 2018), however, the radius of the WD is $9.5 \times 10^{8} \mathrm{~cm}$ and, at a temperature of $15000 \mathrm{~K}$, the implied mass of $0.57 M_{\odot}$ is consistent with a carbon-oxygen interior. Schwope \& Mengel (1997) identified a narrow emission line in EP Dra, which they assigned to the irradiated face of the secondary star. The velocity amplitude $K_{2}^{\prime}=210 \pm 25 \mathrm{~km} \mathrm{~s}^{-1}$, led to a primary mass of $0.43 \pm 0.07 M_{\odot}$. Since the narrow line was not detected over the entire orbit, an independent confirmation is desirable. Hence, with the possible exception of EP Dra, HY Eri may be the only polar with good evidence of a low-mass primary.

A long-standing discrepancy exists between the large number of CVs with low-mass primary stars predicted by binary population synthesis models and the fact that none has been definitely detected so far (e.g., Zorotovic et al. 2011). Schreiber et al. (2016) employed an empirical version of the concept of consequential angular momentum loss (eCAML) that enhances the standard AML caused by magnetic braking and gravitational radiation. The considered CAML is related to nova outbursts and affects primarily low-mass CVs, removing them preferentially from the population. Nelemans et al. (2016) considered asymmetric nova explosions that could provide a kick to the WD and enhanced the mass transfer rate by the ensuing ellipticity of the orbit. Schenker et al. (1998) considered a BondiHoyle type frictional interaction of the secondary with the nova envelope that transfers orbital angular momentum to the shell and may ultimately drive the system over the stability limit. The AML adopted by Schreiber et al. (2016) varies as $C / M_{1}$, with $\mathrm{C}$ a free parameter. For appropriate $C$, it drives low-mass $\mathrm{CVs}$ into catastrophic mass transfer and leaves CVs with massive primaries unaffected. The frictional AML experienced by the secondary star moving in the nova shell is proportional to the ejected mass, which varies approximately as $R_{1}^{4} / M_{1}$. It is furthermore proportional to the duration of the interaction, that is, inversely proportional to the expansion velocity $V_{\text {ex }}$ of the envelope. The mean expansion velocity can exceed $1000 \mathrm{~km} \mathrm{~s}^{-1}$ for 
high-mass WDs, but is much lower for nova events on low-mass WDs, $V_{\text {ex }} \simeq 100-200$ for $0.6 M_{\odot}$ and "a few tens $\mathrm{km} \mathrm{s}^{-1}$ " for $0.4 M_{\odot}$ (Shara et al. 1993). A frictional AML that rises steeply with decreasing mass is, therefore, a plausible proposition. The model may explain the lack of low-mass CVs provided friction proves sufficiently effective or an alternative mechanism as the one of Nelemans et al. (2016) can be identified.

With a mass ratio $q=0.57 \pm 0.06$, HY Eri stays just below the stability limit at $q \simeq 0.65$ (Nelemans et al. 2016; Schreiber et al. 2016), ensuring stable mass transfer in the absence of frictional AML. The added eCAML may render it unstable, if sufficient angular momentum can be extracted from the orbit. The long time scales in low-mass CVs imply, however, that instability is delayed from the time mass transfer started, at least by the waiting time until the first nova outburst and possibly longer. This delay holds similarly for all nova-related CAML descriptions. Nova outbursts in polars with a low-mass primary and typically a rather low accretion rate are especially rare because the ignition mass $\Delta M_{\text {ig }}$ increases (i) with decreasing $\dot{M}$ and (ii) with decreasing $M_{1}$ approximately as $R_{1}^{4} / M_{1}$ (Townsley $\&$ Bildsten 2004, their Fig. 8). For nova outbursts on a He-core WD of $0.4 M_{\odot}$, Shara et al. (1993) found $\Delta M_{\mathrm{ig}} \simeq 9 \times 10^{-4} M_{\odot}$. Such a large ignition mass implies that nova outbursts in HY Eri have a recurrence time in excess of $10^{7} \mathrm{yr}$, given the long-term mean accretion rate of HY Eri of $5 \times 10^{-11} M_{\odot} \mathrm{yr}^{-1}$ (see below). Hence, HY Eri may have accreted already for 10 million years without having experienced a nova outburst that lured it on to destruction. This time span amounts to $\sim 5 \%$ of the typical $2 \times 10^{8} \mathrm{yr}$ it takes non-magnetic CVs to reach the period gap (Knigge et al. 2011). Hence, most CVs with a low-mass primary, must have met their fate much earlier than HY Eri if the eCAML model correctly describes the evolution of CVs. The existence of HY Eri suggests that it is either young and still doomed to destruction or is somehow peculiar, in having managed to escape that outcome.

By its mass, the primary in HY Eri is consistent with a helium WD, but residing in a close binary, it could well be a hybrid star with a mixed He-CO composition (Prada Moroni \& Straniero 2009; Zenati et al. 2019). Such WD are created by massive mass loss of the progenitor star that interrupts He-burning after the star has passed the tip of the RGB. The hydrogendeficient core mass is about $0.46 M_{\odot}$, when stars with an initial mass $M_{i} \lesssim 2 M_{\odot}$ incur the He flash, but for initial masses of 2.2-2.6 $M_{\odot}$, He burning starts at a core mass of $0.32-0.34 M_{\odot}$ and subsequent rapid mass loss in a common envelope event could create a WD with the mass of the primary in HY Eri that has a mixed He-CO composition.

With a period of $2.855 \mathrm{~h}$, HY Eri is nominally located in the 2.1-3.1 h period gap (Knigge et al. 2011), but the high-state accretion rate $\dot{M} \simeq 1.4 \times 10^{-9} M_{\odot} \mathrm{yr}^{-1}$ (Sect. 7.6) demonstrates that HY Eri has not yet entered its period gap, if it ever will. Wickramasinghe \& Wu (1994) and Webbink \& Wickramasinghe (2002) argued that polars experience a lower angular momentum loss by magnetic braking than non-magnetic CVs because trapping of the wind from the secondary star in the WD magnetosphere reduces the braking efficiency. They predicted that the gap disappears for primaries with sufficiently high magnetic moments, $\mu \gtrsim 4 \times 10^{34} \mathrm{Gcm}^{3}$. The WD in HY Eri has $\mu<3.6 \times 10^{34} \mathrm{Gcm}^{3}$, based on $R_{\mathrm{wd}} \simeq 1.22 \times 10^{9} \mathrm{~cm}$ and a polar field strength $B_{\mathrm{p}} \simeq 40 \mathrm{MG}$ of the dipole component (Table 8). This may or may not suffice to suppress magnetic braking and dispose of the gap. Even if HY Eri were about to enter the gap at a period of $2.85 \mathrm{~h}$, the delayed entry compared with the standard upper edge of the gap of $3.1 \mathrm{~h}$ is easily explained by a reduced level of magnetic braking. Given, for example, a braking efficiency reduced by a factor of two over that advocated by Knigge et al. (2011, their Fig. 14, lower panel), the upper edge of the gap would shift down to $2.85 \mathrm{~h}$. A low metalicity of the secondary would add to a delayed entry into the gap (Webbink \& Wickramasinghe 2002). If such a scenario applies to HY Eri, the star may still reside above its own gap and await entering it at some time in the future, if at all.

The telling argument against strong magnetic braking is the low photospheric effective temperature of the WD of $9-10 \mathrm{kK}$. The theory of compressional heating relates $T_{\text {eff }}$ to the mean accretion rate $\langle\dot{M}\rangle$ averaged over the KelvinHelmholtz time scale of the non-degenerate envelope, which is of the order of $10^{6} \mathrm{yr}$ for a low-mass WD (Townsley \& Gänsicke 2009). If the star succeeds in establishing an equilibrium between heating and cooling, its quiescent luminosity is $L_{\mathrm{q}}=6 \times 10^{-3} L_{\odot}\langle\dot{M}\rangle_{-10}\left(M_{1} / M_{\odot}\right)^{0.4}$ (Townsley \& Gänsicke 2009, their Eq. (1)), where $\langle\dot{M}\rangle_{-10}$ is the accretion rate in units of $10^{-10} M_{\odot} \mathrm{yr}^{-1}$. Provided compressional heating dominates over the congenital heat reservoir, we may equate $L_{\mathrm{q}}$ to $4 \pi R_{1}^{2} \sigma T_{\mathrm{ph}}^{4}$. With $R_{1}=6.56 \times 10^{8}\left(M_{1} / M_{\odot}\right)^{-0.60}$ for $M_{1}=0.4-0.6 M_{\odot}$ (Althaus et al. 2013), we obtain $T_{\mathrm{ph}}=16.7\langle\dot{M}\rangle_{-10}^{1 / 4}\left(M_{1} / M_{\odot}\right)^{0.40} \mathrm{kK}$. For $M_{1}=0.42 M_{\odot}$ (Table 10), we find that $\langle\dot{M}\rangle=5 \times 10^{-11} M_{\odot} \mathrm{yr}^{-1}$ suffices to keep the temperature of the WD at $9-10 \mathrm{kK}$. In its high state, HY Eri reached more than $10^{-9} M_{\odot} \mathrm{yr}^{-1}$, implying that the long-term mean duty cycle must be heavily weighted toward states of low accretion. The moderate mass loss of the secondary, the reduced magnetic braking, and the moderate inflation suggested by our best-fit seem to be in line with the evolution of polars as envisaged by Webbink \& Wickramasinghe (2002).

HY Eri experienced a highly significant and so far unexplained change of its orbital period by $10.5 \mathrm{~ms}$ between 2011 and 2018. Similar period variations have been observed in other post common-envelope binaries (PCEB). Attempts to explain the observations involve either (i) the action of additional bodies encircling the binary, causing an apparent period variation, or (ii) solar-cycle like variations in the internal constitution of the secondary star that change its quadrupole moment and the gravitational pull on the primary, leading to a genuine period variation (Applegate 1992; Völschow et al. 2018; Lanza 2020, and references therein). Process (i) is the likely explanation for at least part of the period variations in NN Ser (Beuermann et al. 2013; Bours et al. 2016), but utterly fails in others like QS Vir (Bours et al. 2016), for which no stable planetary model was found, even considering retrograde and highly inclined orbits (S. Dreizler, priv. comm.). The finding of Bours et al. (2016) that PCEB with convective secondaries of spectral type later than M5.5 largely lack period variations seems to favor magnetic cycles as the driving mechanism. The mechanism of Applegate (1992) and the variants of Völschow et al. (2018) and Lanza (2020) are appealing because they are based on physical processes known to exist in late-type stars, but most authors agree that they are too feeble to produce the observed amplitudes of the period variations in many CVs (Völschow et al. 2018; Lanza 2020). It has also been argued that PCEB may be a natural habitat of circumbinary planets (Völschow et al. 2014). The wealth of period variations observed in PCEB and RSCVN binaries may well have more than a single physical cause. The data presently available for HY Eri are not sufficient to draw definite conclusions on the origin of the observed period variation.

In summary, HYEri is a rare example of the polar subgroup of magnetic CVs that harbors a low-mass primary, either a helium WD or a hybrid He-CO WD. The system may have 
K. Beuermann et al.: Neglected X-ray discovered polars. II.

passed through the common-envelope phase after severe mass loss on the giant branch or during initial He-burning (Prada Moroni \& Straniero 2009; Zenati et al. 2019). The key experiment to prove the low mass of the WD would be a direct measurement of its radius. Unfortunately, our Sloan griz photometry of 2017 lacked the time resolution to measure the radius of the WD from the finite ingress and egress times of the eclipse light curves in $g$ or possibly $r$. With HY Eri still in a prolonged state of low accretion as of early 2019 , this task could be accomplished by high-speed photometry at a large telescope. If the low mass of the WD is confirmed, a dedicated evolutionary study could establish the origin and future evolution of HY Eri.

Acknowledgements. We dedicate this paper to the lasting memory of HansChristoph Thomas, who analyzed part of the early data described here before his untimely death on 18 January 2012 . We thank the anonymous referee for the careful reading of the paper and valuable comments that helped to improve this work. Part of the data were collected with the telescopes of the MOnitoring NEtwork of Telescopes, funded by the Alfried Krupp von Bohlen und Halbach Foundation, Essen, and operated by the Georg-August-Universität Göttingen, the McDonald Observatory of the University of Texas at Austin, and the South African Astronomical Observatory. We made use of the ROSAT Data Archive of the Max-Planck-Institut für Extraterrestrische Physik (MPE) at Garching, Germany. Part of the analysis is also based on observations of RE J0501-03 obtained with XMM-Newton on 2002-03-24, Obs-Id 0109460601. The observations at ESO in the years 2000, 2001, 2008, 2016, 2017, and 2018 were collected at the La Silla and Paranal sites under the programme IDs 66.D 0128, 66.D-0513, 082.D-0695, 098.A-9099, and 0100.A-9099. In establishing the spectral energy distribution in Fig. 11, we accessed various data archives via the VizieR Photometric viewer operated at the CDS, Strasbourg, France (http: //vizier.u-strasbg.fr/vizier/sed/). We quoted distances for HY Er and EP Dra from the European Space Agency (ESA) mission Gaia (https: //www.cosmos.esa.int/gaia).

\section{References}

Applegate, J. H. 1992, ApJ, 385, 621

Althaus, L. G., Miller Bertolami, M. M., \& Córsico, A. H. 2013, A\&A, 557, A19

Bailer-Jones, C. A. L., Rybizki, J., Fouesneau, M., Mantelet, G., \& Andrae, R. 2018, AJ, 156, 58

Baraffe, I., Chabrier, G., Allard, F., \& Hauschildt, P. H. 1998, A\&A, 337, 403

Baraffe, I., Homeier, D., Allard, F., \& Chabrier, G. 2015, A\&A, 577, 42

Beuermann, K., \& Reinsch, K. 2008, A\&A, 480, 199

Beuermann, K., \& Thomas, H.-C. 1990, A\&A, 230, 326

Beuermann, K., \& Thomas, H.-C. 1993, Adv. Space Res., 13, 115

Beuermann, K., Thomas, H.-C., Reinsch, K., et al. 1999, A\&A, 347, 47

Beuermann, K., Euchner, F., Reinsch, K., Jordan, S., \& Gänsicke, B. T. 2007, A\&A, 463, 647

Beuermann, K., Burwitz, V., \& Reinsch, K. 2012, A\&A, 543, A41

Beuermann, K., Dreizler, S., \& Hessman, F. V. 2013, A\&A, 555, A133

Beuermann, K., Burwitz, V., Reinsch, K., Schwope, A., \& Thomas, H.-C. 2017, A\&A, 603, A47

Bianchi, L., Efremova, B., Herald, J., et al. 2011, MNRAS, 411, 2770

Boller, T., Freyberg, M. J., Trümper, J., et al. 2016, A\&A, 588, A103

Bonnet-Bidaud, J. M., Mouchet, M., Shakhovskoy, N. M., et al. 2000, A\&A, 354, 1003

Bonnet-Bidaud, J. M., Mouchet, M., Busschaert, C., Falize, E., \& Michaut, C. 2015, A\&A, 579, A24

Bours, M. C. P., Marsh, T. R., Parsons, S. G., et al. 2016, MNRAS, 460, 3873

Burwitz, V., Reinsch, K., Beuermann, K., \& Thomas, H.-C. 1999, Annap. Workshop Magn. Catacl. Var., 157, 127

Busschaert, C., Falize, É., Michaut, C., Bonnet-Bidaud, J.-M., \& Mouchet, M. 2015, A\&A, 579, A25

Chabrier, G., Gallardo, J., \& Baraffe, I. 2007, A\&A, 472, L17

Chanmugam, G., \& Dulk, G. A. 1981, ApJ, 244, 569

Cutri, R. M., Wright, E. L., Conrow, T., et al. 2013, Explanatory Supplement to the AllWISE Data Release Products
Eastman, J., Siverd, R., \& Gaudi, B. S. 2010, PASP, 122, 935

Euchner, F., Jordan, S., Beuermann, K., Gänsicke, B. T., \& Hessman, F. V. 2002, A\&A, 390, 633

Euchner, F., Reinsch, K., Jordan, S., Beuermann, K., \& Gänsicke, B. T. 2005, A\&A, 442, 651

Euchner, F., Jordan, S., Beuermann, K., Reinsch, K., \& Gänsicke, B. T. 2006, A\&A, 451, 671

Ferrario, L., de Martino, D., \& Gänsicke, B. T. 2015, Space Sci. Rev., 191, 111

Fischer, A., \& Beuermann, K. 2001, A\&A, 373, 211

Gaia Collaboration (Brown, A. G. A., et al.) 2018, A\&A, 616, A1

Gänsicke, B. T., Beuermann, K., de Martino, D., \& Thomas, H.-C. 2000, A\&A, 354,605

Hi4PI Collaboration (Ben Bekhti, N., et al.) 2016, VizieR Online Data Catalog: $\mathrm{J} / \mathrm{A}+\mathrm{A} / 594 / \mathrm{A} 116$

Jones, D. O., West, A. A., \& Foster, J. B. 2011, AJ, 142, 44

Jordan, S. 1992, A\&A, 265, 570

Kalirai, J. S., Hansen, B. M. S., Kelson, D. D., et al. 2008, ApJ, 676, 594

König, M., Beuermann, K., \& Gänsicke, B. T. 2006, A\&A, 449, 1129

Knigge, C., Baraffe, I., \& Patterson, J. 2011, ApJS, 194, 28

Kopal, Z. 1959, Close Binary Systems (London: Chapman-Hall and John Wiley), 135

Krzeminski, W., \& Serkowski, K. 1977, ApJ, 216, L45

Lanza, A. F. 2020, MNRAS, 491, 1820

Liebert, J., Wickramasinghe, D. T., Schmidt, G. D., et al. 2005, AJ, 129, 2376

Mann, A. W., Feiden, G. A., Gaidos, E., Boyajian, T., \& von Braun, K. 2015 ApJ, 804, 64

Marsh, T. R., \& Schwope, A. D. 2016, in Astronomy at High Angular Resolution, Astrophys. Space Sci. Lib., 439, 195

Martin, D. C., Fanson, J., Schiminovich, D., et al. 2005, ApJ, 619, L1

Moehler, S., Koester, D., Zoccali, M., et al. 2004, A\&A, 420, 515

Morales, J. C., Gallardo, J., Ribas, I., et al. 2010, ApJ, 718, 502

Nelemans, G., Siess, L., Repetto, S., et al. 2016, ApJ, 817, 69

Panei, J. A., Althaus, L. G., Chen, X., \& Han, Z. 2007, MNRAS, 382, 779

Parsons, S. G., Gänsicke, B. T., Marsh, T. R., et al. 2018, MNRAS, 481, 1083

Prada Moroni, P. G., \& Straniero, O. 2009, A\&A, 507, 1575

Predehl, P., \& Schmitt, J. H. M. M. 1995, A\&A, 293, 889

Putney, A., \& Jordan, S. 1995, ApJ, 449, 863

Renedo, I., Althaus, L. G., Miller Bertolami, M., et al. 2010, ApJ, 717, 183

Renvoizé, V., Baraffe, I., Kolb, U., \& Ritter, H. 2002, A\&A, 389, 485

Ritter, H., \& Kolb, U. 2003, A\&A, 404, 301 (final edition RKcat7.24, 2016)

Roeser, S., Demleitner, M., \& Schilbach, E. 2010, AJ, 139, 2440

Shara, M. M., Prialnik, D., \& Kovetz, A. 1993, ApJ, 406, 220

Schenker, K., Kolb, U., \& Ritter, H. 1998, MNRAS, 297, 633

Schlegel, D. J., Finkbeiner, D. P., \& Davis, M. 1998, ApJ, 500, 525

Schreiber, M. R., Zorotovic, M., \& Wijnen, T. P. G. 2016, MNRAS, 455, L16

Schwope, A. D., \& Christensen, L. 2010, A\&A, 514, A89

Schwope, A. D., \& Mengel, S. 1997, Astron. Nachr., 318, 25

Schwope, A. D., Catalán, M. S., Beuermann, K., et al. 2000, MNRAS, 313, 533

Schwope, A. D., Brunner, H., Buckley, D., et al. 2002, A\&A, 396, 895

Schwope, A. D., Horne, K., Steeghs, D., et al. 2011, A\&A, 531, A34

Skrutskie, M. F., Cutri, R. M., Stiening, R., et al. 2006, AJ, 131, 1163

Spruit, H. C. 1998, ArXiv e-prints [arXiv:astro-ph/9806141]

Staude, A., Schwope, A. D., Hedelt, P., et al. 2004, IAU Colloq. 190: Magnetic Cataclysmic Variables, 251

Thomas, H.-C., Beuermann, K., Reinsch, K., et al. 1998, A\&A, 335, 467

Townsley, D. M., \& Bildsten, L. 2004, ApJ, 600, 390

Townsley, D. M., \& Gänsicke, B. T. 2009, ApJ, 693, 1007

Völschow, M., Banerjee, R., \& Hessman, F. V. 2014, A\&A, 562, A19

Völschow, M., Schleicher, D. R. G., Banerjee, R., \& Schmitt, J. H. M. M. 2018, A\&A, 620, A42

Webbink, R. F., \& Wickramasinghe, D. T. 2002, MNRAS, 335, 1

Wickramasinghe, D. T., \& Wu, K. 1994, MNRAS, 266, L1

Wickramasinghe, D. T., Tout, C. A., \& Ferrario, L. 2014, MNRAS, 437, 675

Woelk, U., \& Beuermann, K. 1992, A\&A, 256, 498

Zacharias, N., Monet, D. G., Levine, S. E., et al. 2005, VizieR Online Data Catalog: I/297

Zenati, Y., Toonen, S., \& Perets, H. B. 2019, MNRAS, 482, 1135

Zorotovic, M., Schreiber, M. R., \& Gänsicke, B. T. 2011, A\&A, 536, A42 